\title{
Nash implementable domains for the Borda count ${ }^{*}$
}

Clemens Puppe ${ }^{1}$ and Attila Tasnádi ${ }^{2}$

${ }^{1}$ Department of Economics, University of Karlsruhe, D - 76128 Karlsruhe, Germany, puppe@wior.uni-karlsruhe.de

2 Department of Mathematics, Corvinus University of Budapest, H - 1093 Budapest, Fővám tér 8, Hungary, attila.tasnadi@uni-corvinus.hu (corresponding author)

Revised Version: November 2007

Appeared in Social Choice and Welfare 31(2008), 367-392.

(C)Springer-Verlag

The original article is available at www.springerlink.com.

DOI: $10.1007 / \mathrm{s} 00355-007-0286-4$

${ }^{*}$ We thank József Mala for posing the question of Nash implementability on restricted domains that led to this research. We are very grateful to two anonymous referees and an associate editor for their helpful comments and suggestions. The second author gratefully acknowledges financial support from the Hungarian Academy of Sciences (MTA) through the Bolyai János research fellowship. 
Summary. We characterize the preference domains on which the Borda count satisfies Maskin monotonicity. The basic concept is the notion of a "cyclic permutation domain" which arises by fixing one particular ordering of alternatives and including all its cyclic permutations. The cyclic permutation domains are exactly the maximal domains on which the Borda count is strategy-proof when combined with every possible tie breaking rule. It turns out that the Borda count is monotonic on a larger class of domains. We show that the maximal domains on which the Borda count satisfies Maskin monotonicity are the "cyclically nested permutation domains" which are obtained from the cyclic permutation domains in an appropriately specified recursive way.

Keywords: Maskin monotonicity, Borda count, restricted preference domains

JEL Classification Number: D71 


\section{Introduction}

A social choice correspondence satisfies Maskin monotonicity if and only if a chosen alternative remains a possible choice whenever in no individual's ranking its relative position to another alternative decreases. It is well-known that Maskin monotonicity, which we shall henceforth refer to simply as "monotonicity," is a necessary condition for Nash implementability; moreover, combined with a no veto power condition it is also sufficient provided that there are at least three individuals (Maskin (1999/1977)). In this paper, we characterize the preference domains on which the Borda count satisfies monotonicity. Since the Borda count satisfies the no veto power condition whenever there are sufficiently many voters, the result thus also yields the preference domains on which the Borda count is Nash implementable.

The celebrated Muller-Satterthwaite theorem (Muller and Satterthwaite (1977)) establishes that, for social choice functions (i.e. single-valued social choice correspondences), monotonicity is equivalent to strategy-proofness, provided that all preference profiles are admissible. By contrast, while strategy-proofness always implies monotonicity, the converse need not be true on restricted domains. In fact, the main result of the present paper provides an illustration of this, showing that there exist preference domains on which the Borda count is monotonic but not strategy-proof when combined with a tie breaking rule.

The preference domains on which the Borda count (with tie breaking) is strategyproof have been characterized in the companion paper Barbie, Puppe and Tasnádi (2006). There, we have shown that, if all individuals face the same domain restriction, the maximal strategy-proof domains for the Borda count are obtained by fixing one particular ordering of the alternatives and including all its cyclic permutations. We refer to such domains as cyclic permutation domains. Here, we show that monotonicity of the Borda count imposes weaker restrictions and allows one to construct domains on which possibility results emerge in a recursive way from the cyclic permutation domains. The corresponding domains are referred to as cyclically nested permutation domains. Specifically, we prove that, under a mild richness condition, the cyclically nested permutation domains are exactly the domains on which the Borda count is monotonic, maintaining the assumption that all individuals face the same domain restriction.

Cyclically nested permutation domains have a more complicated structure than the cyclic permutation domains from which they are recursively constructed. This is the price to be paid when moving from the stronger condition of strategy-proofness to the less demanding condition of monotonicity. In the context of the Borda count, however, monotonicity is a particulary appealing condition since it can be defined in a natural way for social choice correspondences. By contrast, the standard definition of strategyproofness requires a social choice function. Thus, in order to analyze strategy-proofness, the Borda count has first to be transformed into a social choice function using a tie breaking rule. ${ }^{1}$

Cyclic permutation domains as well as their nested refinements are "small" in the sense that each such domain consists of only as many preference orderings as there are alternatives. More specifically, these domains have the restrictive property that

\footnotetext{
${ }^{1}$ In Barbie, Puppe and Tasnádi (2006), we show that in fact some results do depend on the way ties are broken. Strictly speaking, the above mentioned characterization result asserts that the Borda count is strategy-proof for every tie breaking rule if and only if the underlying domain is a cyclic permutation domain.
} 
for each alternative and each rank there exists exactly one preference ordering in the domain that has the given alternative at the given rank in the ordering (see Lemma 2.1 below). Our main characterization result can thus be viewed as the negative finding that the Borda count can be monotonic only on small domains with a very special additional structure.

There is a large literature on domain restrictions in social choice (see Gaertner (2001) for a state-of-the-art summary). Most contributions in this area, however, have studied majority voting and its generalizations, taking Black's (1948) seminal contribution on the notion of single-peaked preferences as the starting point. Some papers, such as Kalai and Muller (1977) and Kalai and Ritz (1980), have analyzed abstract Arrovian aggregation on restricted domains and obtained characterizations of those domains that admit possibility results. As mentioned above, Barbie, Puppe and Tasnádi (2006) obtained a characterization of the maximal domains on which the Borda count is strategy-proof. In a similar vein, Sanver (2007b) characterized the domains on which the plurality rule is strategy-proof, finding that only "trivial" preference domains qualify.

The closest relatives in the literature to the present paper are Bochet and Storcken (2005) and Sanver (2007a). Sanver (2007a) investigates monotonicity of the plurality rule on restricted domains in a model similar to the one used here and shows that the plurality rule can be monotonic only in trivial cases. To the best of our knowledge, Bochet and Storcken (2005) is the first paper to study Maskin monotonicity on restricted preference domains in the framework of the abstract social choice model. ${ }^{2}$ These authors analyze both maximal strategy-proof and maximal monotonic domains for general social choice functions. However, unlike Sanver (2007a) and the present paper in which every individual faces the same preference restriction, Bochet and Storcken (2005) consider restrictions of the preference domain of exactly one individual. By consequence, the social choice functions found to satisfy the desired properties of strategy-proofness and monotonicity have a very special hierarchical structure and are in fact "almost" dictatorial.

\section{Basic Definitions and Statement of Main Result}

Let $X$ be a finite universe of social states or social alternatives and let $q \geq 2$ be its cardinality. By $\mathcal{P}_{X}$ we denote the set of all strict linear orderings (irreflexive, transitive and total binary relations) on $X$; for simplicity, we will henceforth simply speak of linear orderings, dropping the "strict" qualification. By $\mathcal{P} \subseteq \mathcal{P}_{X}$ we denote a generic subdomain of the unrestricted domain $\mathcal{P}_{X}$.

Definition (Social choice rule) A mapping $f: \bigcup_{n=1}^{\infty} \mathcal{P}^{n} \rightarrow 2^{X} \backslash\{\emptyset\}$ that assigns a set of (most preferred) alternatives $f\left(\succ_{1}, \ldots, \succ_{n}\right) \in 2^{X} \backslash\{\emptyset\}$ to each $n$-tuple of linear orderings and all $n$ is called a social choice rule (SCR).

Let $r k[x, \succ]$ denote the rank of alternative $x$ in the ordering $\succ$ (i.e. $r k[x, \succ]=1$ if $x$ is

\footnotetext{
${ }^{2}$ There is also a more distantly related literature on monotonic extensions of social choice rules. For instance, the work of Erdem and Sanver (2005) is also motivated by the observation that the Borda count, and in fact any scoring method, violates the monotonicity condition on an unrestricted domain. However, the monotonic extensions are again defined on the unrestricted preference domain; therefore, the analysis does not contribute to the question on which preference domains the original (non-extended) social rule would satisfy monotonicity.
} 
the top alternative in the ranking $\succ, r k[x, \succ]=2$ if $x$ is second-best, and so on).

Definition (Borda count) The SCR $f^{B}$ associated with the Borda count is given as follows: for all $n$ and all $\succ_{1}, \ldots, \succ_{n} \in \mathcal{P}_{X}$ we have

$$
x \in f^{B}\left(\succ_{1}, \ldots, \succ_{n}\right) \Leftrightarrow \sum_{i=1}^{n} r k\left[x, \succ_{i}\right] \leq \sum_{i=1}^{n} r k\left[y, \succ_{i}\right] \quad \text { for all } y \in X .
$$

We shall denote by $L(x, \succ)=\{y \in X \mid x \succ y\}$ the lower contour set and by $U(x, \succ)=$ $\{y \in X \mid y \succ x\}$ the upper contour set of the preference $\succ$ at the alternative $x \in X$. A SCR $f$ is called monotonic on $\mathcal{P}$ if for all $x \in X$, all $n$ and all $\succ_{1}, \ldots, \succ_{n}, \succ_{1}^{\prime} \ldots, \succ_{n}^{\prime} \in \mathcal{P}$ we have

$$
\left[x \in f\left(\succ_{1}, \ldots, \succ_{n}\right), L\left(x, \succ_{i}\right) \subseteq L\left(x, \succ_{i}^{\prime}\right) \text { for all } i=1, \ldots, n\right] \Rightarrow x \in f\left(\succ_{1}^{\prime}, \ldots, \succ_{n}^{\prime}\right) .
$$

We call a domain $\mathcal{P}$ Borda monotonic if $f^{B}$ is monotonic on $\mathcal{P}$. Note that any subdomain of a (Borda) monotonic domain is (Borda) monotonic. Given a profile of preferences $\left(\succ_{1}, \ldots, \succ_{n}\right) \in \mathcal{P}^{n}$, we say that alternatives $A \subseteq X$ are indifferent on the top if $A=f^{B}\left(\succ_{1}, \ldots, \succ_{n}\right)$.

We will only be interested in preference domains that are minimally rich since without such condition properties such as monotonicity or strategy-proofness can be satisfied in a trivial way. ${ }^{3}$ Specifically, we will impose the following condition.

Definition (Minimally rich domain) A domain $\mathcal{P}$ is called minimally rich if, for any $x \in X$, there exists (i) $\succ \in \mathcal{P}$ such that $r k[x, \succ]=1$, and (ii) $\succ^{\prime} \in \mathcal{P}$ such that $r k\left[x, \succ^{\prime}\right]=q$.

Thus, our minimal richness condition requires that each alternative must be (i) most preferred by at least one preference ordering, and (ii) least preferred by some (other) preference ordering. This is slightly stronger than the richness condition used in Barbie, Puppe and Tasnádi (2006) which consisted of part (i) only. ${ }^{4}$ Part (ii) of the present condition is needed in Lemma 5.3 and in Substep 2B of the proof of our main result below.

\section{Cyclically nested permutation domains}

An ordering $\succ^{\prime}$ is called a cyclic permutation of $\succ$ if $\succ^{\prime}$ can be obtained from $\succ$ by sequentially shifting the bottom element to the top while leaving the order between all other alternatives unchanged. Thus, for instance, the cyclic permutations of the ordering $a b c d$ are $d a b c, c d a b$ and $b c d a$. The set of all cyclic permutations of a fixed ordering $\succ$ is denoted by $\mathcal{Z}(\succ)$, which we also call a cyclic permutation domain. In Barbie, Puppe and Tasnádi (2006), we have shown that the cyclic permutation domains are exactly the domains on which the Borda count is strategy-proof when combined with any conceivable deterministic tie-breaking rule. ${ }^{5}$ The cyclic permutation domains serve as the building blocks of the so-called "cyclically nested permutation domains"

\footnotetext{
${ }^{3}$ Obviously, every social choice function (i.e. single-valued social choice rule) is strategy-proof and monotonic on any domain consisting of only one preference ordering.

${ }^{4}$ Although even weaker than the "minimal" richness condition used here, the condition in Barbie, Puppe and Tasnádi (2006) is simply called "richness" there.

${ }^{5}$ Combined with particular, appropriately chosen tie-breaking rules the Borda count can be strategy-proof on a larger class of domains, see Barbie, Puppe and Tasnádi (2006).
} 
to be defined presently. The following is our main result.

Theorem A domain is minimally rich and Borda monotonic if and only if it is a cyclically nested permutation domain.

Before giving a formal definition of cyclically nested permutation (henceforth, CNP) domains, we start with an intuitive example illustrating the basic recursive construction of CNP domains. A CNP domain on $q$ alternatives consists of $q$ preferences and can therefore be represented by a matrix that collects the preferences in its columns with the best alternative in the first row, the second-best alternative in the second row, and so on. For instance, the matrix in Table 1 represents a cyclic permutation domain on a set of three alternatives.

Table 1: Initial step

$$
\left(\begin{array}{lll}
a & b & c \\
b & c & a \\
c & a & b
\end{array}\right)
$$

We may now replace the elements of the matrix with different square matrices representing cyclical permutation domains of identical size. For instance, in Table 1 we may replace each element with a $2 \times 2$ matrix to obtain the matrix shown in Table 2. Thus, we have constructed a new CNP domain on a set of six alternatives. This procedure can repeated any finite number of times, replacing at each step the elements of the given matrix with square matrices of identical size storing different cyclical permutation domains. However, in order to remain within the class of admissible CNP domains, the "replacement mechanism" has to be further restricted, as explained below.

Table 2: A new CNP domain

$$
\left(\begin{array}{cc|cc|cc}
d & e & f & g & h & i \\
e & d & g & f & i & h \\
\hline f & g & h & i & d & e \\
g & f & i & h & e & d \\
\hline h & i & d & e & f & g \\
i & h & e & d & g & f
\end{array}\right)
$$

Let us then turn to the formal definition of CNP domains. First, the cyclic permutation domains themselves are called CNP domains of depth 1 . Second, we define CNP domains of depth 2 , as follows. Assume that $q=q_{1} q_{2}$, where $q_{1}, q_{2}$ are two integers greater than 1 . Take an arbitrary partition $X_{1}, \ldots, X_{q_{2}}$ of $X$ into equally sized sets (i.e., $\# X_{i}=q_{1}$ for all $\left.i=1, \ldots, q_{2}\right)$ and let $X^{\prime}=\left\{X_{1}, \ldots, X_{q_{2}}\right\}$. Pick a linear ordering $\succ^{\prime} \in \mathcal{P}_{X^{\prime}}$ and consider the domain $\mathcal{Z}\left(\succ^{\prime}\right)$. We now replace each set $X_{i}$ with a cyclic permutation domain defined on the set of alternatives $X_{i}$ with cardinality $q_{1}$. For example, if $q_{2}=3$ and $q_{1}=2$, we first obtain the domain at the left hand side of Table 3 and thereafter the domain at the right hand side of this table. Note that 
Table 3: Constructing CNP domains

\begin{tabular}{|c|c|c|c|c|c|c|c|c|}
\hline$\succ_{1}^{\prime}$ & $\succ_{2}^{\prime}$ & $\succ_{3}^{\prime}$ & $\succ_{1}$ & $\succ_{2}$ & $\succ 3$ & $\succ_{4}$ & $\succ_{5}$ & $\succ_{6}$ \\
\hline$X_{1}$ & $X_{2}$ & $X_{3}$ & $x_{1}$ & $x_{2}$ & $y_{2}$ & $y_{1}$ & $z_{2}$ & $z_{1}$ \\
\hline$X_{2}$ & $X_{3}$ & $X_{1}$ & $x_{2}$ & $x_{1}$ & $y_{1}$ & $y_{2}$ & $z_{1}$ & $z_{2}$ \\
\hline \multirow[t]{4}{*}{$X_{3}$} & $X_{1}$ & $X_{2}$ & $y_{1}$ & $y_{2}$ & $z_{2}$ & $z_{1}$ & $x_{1}$ & $x_{2}$ \\
\hline & & & $y_{2}$ & $y_{1}$ & $z_{1}$ & $z_{2}$ & $x_{2}$ & $x_{1}$ \\
\hline & & & $z_{1}$ & $z_{2}$ & $x_{1}$ & $x_{2}$ & $y_{1}$ & $y_{2}$ \\
\hline & & & $z_{2}$ & $z_{1}$ & $x_{2}$ & $x_{1}$ & $y_{2}$ & $y_{1}$ \\
\hline
\end{tabular}

each factorization of $q$ into two factors results in different CNP domains of depth 2; moreover, the order of the factors obviously also matters.

To formalize the "replacement" mechanism indicated in Table 3, pick arbitrary linear orderings $\succ_{1}^{*} \in \mathcal{P}_{X_{1}}, \ldots, \succ_{q_{2}}^{*} \in \mathcal{P}_{X_{q_{2}}}$. For each linear ordering $\succ^{\prime \prime} \in \mathcal{Z}\left(\succ^{\prime}\right)$ on $X^{\prime}$ we can construct a set of preferences $\mathcal{P}^{\prime}=\left\{\succ_{1}^{\prime \prime}, \ldots, \succ_{q_{1}}^{\prime \prime}\right\}$ on $X$ such that (i) $\left\{\succ_{1 \mid X_{i}}^{\prime \prime}, \ldots, \succ_{q_{1 \mid X_{i}}}^{\prime \prime}\right\}=\mathcal{Z}\left(\succ_{i}^{*}\right)$, where $\succ_{k \mid X_{i}}^{\prime \prime}$ denotes the restriction of $\succ_{k}^{\prime \prime}$ to $X_{i}$, and (ii) $X_{i} \succ^{\prime \prime} X_{j}$ implies $x \succ_{k}^{\prime \prime} y$ for all $i, j=1, \ldots, q_{2}$, all $x \in X_{i}$, all $y \in X_{j}$ and all $k=1, \ldots, q_{1}$. Observe that by construction any CNP domain of depth 2 on $X$ consists of exactly $q=\# X$ preferences.

However, in order to guarantee monotonicity of the Borda count, we must restrict the admissible replacements by cyclical permutation domains. To see this consider the domain shown in Table 4. Pick a profile $\Pi$ consisting of one voter of each type. Then $f^{B}(\Pi)=\left\{x_{1}, x_{2}, y_{1}, y_{2}, z_{1}, z_{2}\right\}$ and monotonicity is violated at alternative $x_{2}$ if, for instance, the voter of type $\succ_{3}$ switches to type $\succ_{6}$. Indeed, while $x_{2}$ improves by two ranks if the the voter of type $\succ_{3}$ switches to type $\succ_{6}$, the alternative $z_{1}$ even improves by three ranks and in fact becomes the unique Borda winner.

Table 4: A non-monotonic domain

\begin{tabular}{cccccc}
$\succ_{1}$ & $\succ_{2}$ & $\succ_{3}$ & $\succ_{4}$ & $\succ_{5}$ & $\succ_{6}$ \\
\hline$x_{1}$ & $x_{2}$ & $y_{2}$ & $y_{1}$ & $z_{2}$ & $z_{1}$ \\
$x_{2}$ & $x_{1}$ & $y_{1}$ & $y_{2}$ & $z_{1}$ & $z_{2}$ \\
$y_{1}$ & $y_{2}$ & $z_{2}$ & $z_{1}$ & $x_{2}$ & $x_{1}$ \\
$y_{2}$ & $y_{1}$ & $z_{1}$ & $z_{2}$ & $x_{1}$ & $x_{2}$ \\
$z_{1}$ & $z_{2}$ & $x_{1}$ & $x_{2}$ & $y_{1}$ & $y_{2}$ \\
$z_{2}$ & $z_{1}$ & $x_{2}$ & $x_{1}$ & $y_{2}$ & $y_{1}$
\end{tabular}

We restrict the admissible replacements by cyclical permutation domains by specifying a set of ordered pairs $(x, y)$ of alternatives that must have the same rank difference in all preference orderings that rank $x$ above $y$. For example, in Table 3 the rank differences between $x_{1}$ and $y_{1}$ is 2 in all those preferences that rank $x_{1}$ above $y_{1}$; similarly, the rank difference between $x_{1}$ and $z_{1}$ is 4 in all preference orderings that rank $x_{1}$ above $z_{1}$. By contrast, in preference $\succ_{5}$ the alternative $z_{1}$ is one rank above $x_{1}$, while 
it is 3 ranks above in $\succ_{6} \cdot{ }^{6}$ Formally, for a domain to qualify as a CNP domain there must exist, for all $i, j \in\left\{1, \ldots, q_{2}\right\}$ with $i \neq j$, bijections $\varphi_{i, j}: X_{i} \rightarrow X_{j}$ such that, for all $x \in X_{i}$, the rank difference between $x$ and $\varphi_{i, j}(x)$ is the same in all preference orderings that rank $x$ above $\varphi_{i, j}(x)$. To illustrate, consider again the CNP domain on the right hand side of Table 3 . As is easily verified the required bijections exist in that case; for instance, $\varphi_{1,3}\left(x_{1}\right)=z_{1}, \varphi_{1,3}\left(x_{2}\right)=z_{2}, \varphi_{3,1}\left(z_{1}\right)=x_{2}$ and $\varphi_{3,1}\left(z_{2}\right)=x_{1}{ }^{7}$ By contrast, for the domain shown in Table 4 there exists no bijection with the required properties between the sets $X_{3}=\left\{z_{1}, z_{2}\right\}$ and $X_{1}=\left\{x_{1}, x_{2}\right\}$, for instance.

Now assume that we have defined all CNP domains of depth $n-1$ and let $q=\prod_{i=1}^{n} q_{i}$ where $q_{1}, \ldots, q_{n}$ are integers greater than 1 . The class of CNP domains of depth $n$ is defined as follows. Take an arbitrary partition $X_{1}, \ldots, X_{q_{n}}$ of $X$ into equally sized sets (i.e., $q^{\prime}=\# X_{i}=q / q_{n}$ for all $\left.i=1, \ldots q_{n}\right)$ and let $X^{\prime}=\left\{X_{1}, \ldots, X_{q_{n}}\right\}$. Pick arbitrary CNP domains $\mathcal{P}_{1} \subseteq \mathcal{P}_{X_{1}}, \ldots, \mathcal{P}_{q_{n}} \subseteq \mathcal{P}_{X_{q_{n}}}$ with the associated factorization $\prod_{i=1}^{n-1} q_{i}$, a linear ordering $\succ^{\prime} \in \mathcal{P}_{X^{\prime}}$, and consider the domain $\mathcal{Z}\left(\succ^{\prime}\right)$. For each linear ordering $\succ^{\prime \prime} \in \mathcal{Z}\left(\succ^{\prime}\right)$ on $X^{\prime}$ construct a set of preferences $\mathcal{P}^{\prime}=\left\{\succ_{1}^{\prime \prime}, \ldots, \succ_{q^{\prime}}^{\prime \prime}\right\}$ on $X$ such that (i) $\left\{\succ_{1 \mid X_{i}}^{\prime \prime}, \ldots, \succ_{q^{\prime} \mid X_{i}}^{\prime \prime}\right\}=\mathcal{P}_{i}$, (ii) $X_{i} \succ^{\prime \prime} X_{j}$ implies $x \succ_{k}^{\prime \prime} y$ for all $i, j=1, \ldots, q_{n}$, all $x \in X_{i}$, all $y \in X_{j}$ and all $k=1, \ldots, q^{\prime}$, and (iii) there exist, for all $i, j \in\left\{1, \ldots, q_{n}\right\}$ with $i \neq j$, bijections $\varphi_{i, j}: X_{i} \rightarrow X_{j}$ such that, for all $x \in X_{i}, x$ and $\varphi_{i, j}(x)$ have the same rank differences in all preferences of the form $\succ_{k}^{\prime \prime}$ that rank $x$ above $\varphi_{i, j}(x)$, for all $\succ^{\prime \prime} \in \mathcal{Z}\left(\succ^{\prime}\right)$ and all $k$.

Observe that, by construction, any CNP domain on $X$ consists of exactly $q=\# X$ preferences. Furthermore, one can easily determine the maximal depth of a CNP domain on a given number of alternatives, as follows. Suppose that the prime factorization of $q$ takes the form $q=\prod_{i=1}^{k} p_{i}^{m_{i}}$, where $p_{i}$ are primes and $m_{i}$ are positive integers for all $i=1, \ldots, k$; moreover, let $D_{q}:=\sum_{i=1}^{k} m_{i}$. Then, the maximal depth of a CNP domain on $q$ alternatives is $D_{q}$. In particular, if $q$ is a prime, only the cyclic permutation domains themselves qualify as CNP domains.

We provide an example of a CNP of depth 3 with $q_{1}=2, q_{2}=3$ and $q_{3}=2$ to further illustrate the definition of CNP domains. The first domain is a cyclical permutation domain defined on two sets of alternatives as shown in Table 5 . Let

Table 5: Initial step

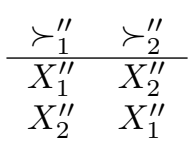

$X_{1}^{\prime \prime}=\left\{x_{1}, x_{2}, \ldots, x_{6}\right\}, X_{2}^{\prime \prime}=\left\{x_{7}, x_{8}, \ldots, x_{12}\right\}, \varphi_{1,2}\left(x_{i}\right)=x_{i+6}$ for $i=1, \ldots, 6$, and $\varphi_{2,1}=\varphi_{1,2}^{-1}$. Next, we replace each set $X_{1}^{\prime \prime}$ and $X_{2}^{\prime \prime}$ with a CNP domain of depth 2 and associated factorization $2 \cdot 3$. We derive these two CNP domains simultaneously in Table 6. Furthermore, we select the partition $X_{1}^{\prime}=\left\{x_{1}, x_{2}\right\}, X_{2}^{\prime}=\left\{x_{3}, x_{4}\right\}, X_{3}^{\prime}=\left\{x_{5}, x_{6}\right\}$ of $X_{1}^{\prime \prime}$ and the partition $X_{4}^{\prime}=\left\{x_{7}, x_{8}\right\}, X_{5}^{\prime}=\left\{x_{9}, x_{10}\right\}, X_{6}^{\prime}=\left\{x_{11}, x_{12}\right\}$ of $X_{2}^{\prime \prime}$.

\footnotetext{
${ }^{6}$ Note that this can happen only across different sets $X_{i}$ and $X_{j}$; indeed, within the sets $X_{i}$, and generally in any cyclical permutation domain, two alternatives that have the same relative position in two different preference orderings must also have the same rank difference.

${ }^{7}$ Note, in particular, that we do not necessarily require $\varphi_{i, j}=\varphi_{j, i}^{-1}$.
} 
Table 6: Constructing the CNP domains on $X_{1}^{\prime \prime}$ and $X_{2}^{\prime \prime}$

\begin{tabular}{ccccccc}
$\succ_{1}^{\prime}$ & $\succ_{2}^{\prime}$ & $\succ_{3}^{\prime}$ & & $\succ_{4}^{\prime}$ & $\succ_{5}^{\prime}$ & $\succ_{6}^{\prime}$ \\
\hline$X_{1}^{\prime}$ & $X_{2}^{\prime}$ & $X_{3}^{\prime}$ & & $X_{4}^{\prime}$ & $X_{5}^{\prime}$ & $X_{6}^{\prime}$ \\
$X_{2}^{\prime}$ & $X_{3}^{\prime}$ & $X_{1}^{\prime}$ & & $X_{5}^{\prime}$ & $X_{6}^{\prime}$ & $X_{4}^{\prime}$ \\
$X_{3}^{\prime}$ & $X_{1}^{\prime}$ & $X_{2}^{\prime}$ & & $X_{6}^{\prime}$ & $X_{4}^{\prime}$ & $X_{5}^{\prime}$
\end{tabular}

The sets $X_{1}^{\prime}, \ldots, X_{6}^{\prime}$ are then replaced by cyclical permutation domains each defined on the respective sets of two alternatives. Specifically, we replace $X_{i}$ by $\mathcal{Z}\left(x_{2 i-1} \succ x_{2 i}\right)$ for all $i=1, \ldots, 6$, and we choose bijections such that $\varphi_{1,2}^{\prime}\left(x_{1}\right)=x_{3}, \varphi_{1,2}^{\prime}\left(x_{2}\right)=x_{4}$, $\varphi_{1,3}^{\prime}\left(x_{1}\right)=x_{5}, \varphi_{1,3}^{\prime}\left(x_{2}\right)=x_{6}, \varphi_{2,3}^{\prime}\left(x_{3}\right)=x_{5}, \varphi_{2,3}^{\prime}\left(x_{4}\right)=x_{6}, \varphi_{2,1}^{\prime}=\left(\varphi_{1,2}^{\prime}\right)^{-1}, \varphi_{3,1}^{\prime}=$ $\left(\varphi_{1,3}^{\prime}\right)^{-1}, \varphi_{3,2}^{\prime}=\left(\varphi_{2,3}^{\prime}\right)^{-1}$. We thus obtain the CNP domain shown at the left hand side of Table 7. In an analogous way, we construct the CNP domain shown at the right hand side of Table 7. Finally, inserting the two CNP domains of Table 7 into Table 5 and employing the bijections $\varphi_{1,2}$ and $\varphi_{2,1}$, we obtain the CNP domain of depth 3 shown in Table 8.

Table 7: The CNP domains on $X_{1}^{\prime \prime}$ and $X_{2}^{\prime \prime}$

\begin{tabular}{cccccc}
$\succ_{1}^{*}$ & $\succ_{2}^{*}$ & $\succ_{3}^{*}$ & $\succ_{4}^{*}$ & $\succ_{5}^{*}$ & $\succ_{6}^{*}$ \\
\hline$x_{1}$ & $x_{2}$ & $x_{3}$ & $x_{4}$ & $x_{5}$ & $x_{6}$ \\
$x_{2}$ & $x_{1}$ & $x_{4}$ & $x_{3}$ & $x_{6}$ & $x_{5}$ \\
$x_{3}$ & $x_{4}$ & $x_{5}$ & $x_{6}$ & $x_{1}$ & $x_{2}$ \\
$x_{4}$ & $x_{3}$ & $x_{6}$ & $x_{5}$ & $x_{2}$ & $x_{1}$ \\
$x_{5}$ & $x_{6}$ & $x_{1}$ & $x_{2}$ & $x_{3}$ & $x_{4}$ \\
$x_{6}$ & $x_{5}$ & $x_{2}$ & $x_{1}$ & $x_{4}$ & $x_{3}$
\end{tabular}

\begin{tabular}{cccccc}
$\succ_{7}^{*}$ & $\succ_{8}^{*}$ & $\succ_{9}^{*}$ & $\succ_{10}^{*}$ & $\succ_{11}^{*}$ & $\succ_{12}^{*}$ \\
\hline$x_{7}$ & $x_{8}$ & $x_{9}$ & $x_{10}$ & $x_{11}$ & $x_{12}$ \\
$x_{8}$ & $x_{7}$ & $x_{10}$ & $x_{9}$ & $x_{12}$ & $x_{11}$ \\
$x_{9}$ & $x_{10}$ & $x_{11}$ & $x_{12}$ & $x_{7}$ & $x_{8}$ \\
$x_{10}$ & $x_{9}$ & $x_{12}$ & $x_{11}$ & $x_{8}$ & $x_{7}$ \\
$x_{11}$ & $x_{12}$ & $x_{7}$ & $x_{8}$ & $x_{9}$ & $x_{10}$ \\
$x_{12}$ & $x_{11}$ & $x_{8}$ & $x_{7}$ & $x_{10}$ & $x_{9}$
\end{tabular}

We conclude this section with a simple necessary condition for a domain to qualify as a CNP domain.

Lemma 2.1 Any $C N P$ domain $\mathcal{P}$ on $X$ consists of exactly $q$ preferences, and for all $x \in X$ and all $i \in\{1, \ldots, q\}$ there exists exactly one preference $\succ \in \mathcal{P}$ such that $r k[x, \succ]=i$.

Proof of Lemma 2.1 The statement can be established by induction on the depth of CNP domains. Cyclical permutation domains clearly satisfy the stated property. Assume that the statement holds for all CNP domains of depth $n-1$. Take a CNP domain $\mathcal{P}$ of depth $n$ that is constructed from a cyclical permutation domain on $X^{\prime}=\left\{X_{1}, \ldots, X_{q_{n}}\right\}$ (where the $X_{i}$ are sets of equal size) and from CNP domains of depth $n-1$ replacing each set $X_{i}$. The stated property follows at once from the induction hypothesis for the CNP domains of depth $n-1$ and the structure of a cyclical permutation domain.

Note that, while any subdomain of a CNP domain is Borda monotonic, no proper subdomain of a CNP domain can be minimally rich by Lemma 2.1. Moreover, we have 
Table 8: A CNP domain of depth 3

\begin{tabular}{cccccccccccc}
$\succ_{1}$ & $\succ_{2}$ & $\succ_{3}$ & $\succ_{4}$ & $\succ_{5}$ & $\succ_{6}$ & $\succ_{7}$ & $\succ_{8}$ & $\succ_{9}$ & $\succ_{10}$ & $\succ_{11}$ & $\succ_{12}$ \\
\hline$x_{1}$ & $x_{2}$ & $x_{3}$ & $x_{4}$ & $x_{5}$ & $x_{6}$ & $x_{7}$ & $x_{8}$ & $x_{9}$ & $x_{10}$ & $x_{11}$ & $x_{12}$ \\
$x_{2}$ & $x_{1}$ & $x_{4}$ & $x_{3}$ & $x_{6}$ & $x_{5}$ & $x_{8}$ & $x_{7}$ & $x_{10}$ & $x_{9}$ & $x_{12}$ & $x_{11}$ \\
$x_{3}$ & $x_{4}$ & $x_{5}$ & $x_{6}$ & $x_{1}$ & $x_{2}$ & $x_{9}$ & $x_{10}$ & $x_{11}$ & $x_{12}$ & $x_{7}$ & $x_{8}$ \\
$x_{4}$ & $x_{3}$ & $x_{6}$ & $x_{5}$ & $x_{2}$ & $x_{1}$ & $x_{10}$ & $x_{9}$ & $x_{12}$ & $x_{11}$ & $x_{8}$ & $x_{7}$ \\
$x_{5}$ & $x_{6}$ & $x_{1}$ & $x_{2}$ & $x_{3}$ & $x_{4}$ & $x_{11}$ & $x_{12}$ & $x_{7}$ & $x_{8}$ & $x_{9}$ & $x_{10}$ \\
$x_{6}$ & $x_{5}$ & $x_{2}$ & $x_{1}$ & $x_{4}$ & $x_{3}$ & $x_{12}$ & $x_{11}$ & $x_{8}$ & $x_{7}$ & $x_{10}$ & $x_{9}$ \\
$x_{7}$ & $x_{8}$ & $x_{9}$ & $x_{10}$ & $x_{11}$ & $x_{12}$ & $x_{1}$ & $x_{2}$ & $x_{3}$ & $x_{4}$ & $x_{5}$ & $x_{6}$ \\
$x_{8}$ & $x_{7}$ & $x_{10}$ & $x_{9}$ & $x_{12}$ & $x_{11}$ & $x_{2}$ & $x_{1}$ & $x_{4}$ & $x_{3}$ & $x_{6}$ & $x_{5}$ \\
$x_{9}$ & $x_{10}$ & $x_{11}$ & $x_{12}$ & $x_{7}$ & $x_{8}$ & $x_{3}$ & $x_{4}$ & $x_{5}$ & $x_{6}$ & $x_{1}$ & $x_{2}$ \\
$x_{10}$ & $x_{9}$ & $x_{12}$ & $x_{11}$ & $x_{8}$ & $x_{7}$ & $x_{4}$ & $x_{3}$ & $x_{6}$ & $x_{5}$ & $x_{2}$ & $x_{1}$ \\
$x_{11}$ & $x_{12}$ & $x_{7}$ & $x_{8}$ & $x_{9}$ & $x_{10}$ & $x_{5}$ & $x_{6}$ & $x_{1}$ & $x_{2}$ & $x_{3}$ & $x_{4}$ \\
$x_{12}$ & $x_{11}$ & $x_{8}$ & $x_{7}$ & $x_{10}$ & $x_{9}$ & $x_{6}$ & $x_{5}$ & $x_{2}$ & $x_{1}$ & $x_{4}$ & $x_{3}$
\end{tabular}

the following corollary.

Corollary 1 If $\mathcal{P}=\left\{\succ_{1}, \ldots, \succ_{q}\right\}$ is a CNP domain on $X$, then $f^{B}\left(\succ_{1}, \ldots, \succ_{q}\right)=X$, i.e. all alternatives are indifferent on the top if each preference occurs exactly once in a profile.

\section{Characterizing and Detecting CNP Domains}

We characterized cyclical permutation domains in Barbie, Puppe and Tasnádi (2006) by the equal rank difference condition, which we recall here for minimally rich domains.

Definition (Equal rank difference) A minimally rich domain $\mathcal{P}$ satisfies the equal rank difference (henceforth, ERD) condition if for all $x, y \in X$, all $\succ, \succ^{\prime} \in \mathcal{P}$

$$
\left(x \succ y \text { and } x \succ^{\prime} y\right) \Rightarrow r k[x, \succ]-r k[y, \succ]=r k\left[x, \succ^{\prime}\right]-r k\left[y, \succ^{\prime}\right] .
$$

Considering the restricted rank difference condition associated with the bijections $\varphi_{i, j}$ : $X_{i} \rightarrow X_{j}$ in the recursive definition of CNP domains, an appropriate weakening of the ERD condition leads to a characterization of CNP domains.

Definition (Nested equal rank difference) A domain $\mathcal{P}$ satisfies the nested equal rank difference (henceforth, NERD) condition if there exists a "nested" set system

$$
\left\{X, X_{i_{n}}, X_{i_{n-1}, i_{n}}, \ldots, X_{i_{2}, \ldots, i_{n-1}, i_{n}}, X_{i_{1}, i_{2}, \ldots, i_{n-1}, i_{n}}\right\}_{i_{n}=1, \ldots, i_{1}=1}^{q_{n}, \ldots, q_{1}}
$$

on $X$ such that

1. $q=\prod_{i=1}^{n} q_{i}$, where $q_{i}$ are integers greater than 1 for all $i=1, \ldots, n$;

2. $X=\cup_{i=1}^{q_{n}} X_{i}, \# X_{i}=q / q_{n}$ for all $i=1, \ldots, q_{n}$;

(a) for all $\succ \in \mathcal{P}$, all $x \in X_{i}$ and all $y \in X_{j}$ we have that $x \succ y$ implies $x^{\prime} \succ y^{\prime}$ for all $x^{\prime} \in X_{i}$ and all $y^{\prime} \in X_{j}$; 
(b) there exist bijections $\varphi_{i, j}^{n}: X_{i} \rightarrow X_{j}$ for all $i, j=1, \ldots, q_{n}, i \neq j$ such that

$$
x \succ y=\varphi_{i, j}^{n}(x) \text { and } x \succ^{\prime} y \Rightarrow r k[x, \succ]-r k[y, \succ]=r k\left[x, \succ^{\prime}\right]-r k\left[y, \succ^{\prime}\right]
$$

for all $x \in X_{i}$ and all $\succ, \succ^{\prime} \in \mathcal{P}$;

3. for all $l=2, \ldots, n$ and all $\left(i_{l}, i_{l+1}, \ldots, i_{n}\right) \in \times_{k=l}^{n}\left\{1, \ldots, q_{k}\right\}$ we have

(a) $X_{i_{l}, i_{l+1}, \ldots, i_{n-1}, i_{n}}=\cup_{i_{l-1}=1}^{q_{l-1}} X_{i_{l-1}, i_{l}, \ldots, i_{n-1}, i_{n}}$,

$\# X_{i_{l-1}, i_{l}, \ldots, i_{n-1}, i_{n}}=\prod_{k=1}^{l-2} q_{k}$ for all $i_{l-1}=1, \ldots, q_{l-1} ;$

(b) for all $\succ \in \mathcal{P}$, all $i, j=1, \ldots, q_{l-1}, i \neq j$, all $x \in X_{i, i_{l}, \ldots, i_{n-1}, i_{n}}$ and all $y \in$ $X_{j, i_{l}, \ldots, i_{n-1}, i_{n}}$ we have that $x \succ y$ implies $x^{\prime} \succ y^{\prime}$ for all $x^{\prime} \in X_{i, i_{l}, \ldots, i_{n-1}, i_{n}}$ and $y^{\prime} \in X_{j, i_{l}, \ldots, i_{n-1}, i_{n}} ;$

(c) there exists bijections $\varphi_{i, j}^{l-1}: X_{i, i_{l}, \ldots, i_{n-1}, i_{n}} \rightarrow X_{j, i_{l}, \ldots, i_{n-1}, i_{n}}$ for all $i, j=$ $1, \ldots, q_{l-1}, i \neq j$ such that

$$
x \succ y=\varphi_{i, j}^{l-1}(x) \text { and } x \succ^{\prime} y \Rightarrow r k[x, \succ]-r k[y, \succ]=r k\left[x, \succ^{\prime}\right]-r k\left[y, \succ^{\prime}\right]
$$

for all $x \in X_{i, i_{l}, \ldots, i_{n-1}, i_{n}}$ and all $\succ, \succ^{\prime} \in \mathcal{P}$.

Observe that a minimally rich domain $\mathcal{P}$ satisfies ERD if and only if it satisfies NERD on the nested set system $\left\{X,\left\{x_{1}\right\},\left\{x_{2}\right\}, \ldots,\left\{x_{q}\right\}\right\}$. Thus, ERD is a special case of NERD.

Proposition $1 \mathcal{P}$ is a CNP domain if and only if $\mathcal{P}$ is minimally rich and satisfies NERD.

Proof Assume that $\mathcal{P}$ is a CNP domain. We prove that $\mathcal{P}$ satisfies NERD by induction on the depth of CNP domains. By the analysis of Barbie, Puppe and Tasnádi (2006), cyclic permutation domains satisfy ERD. Thus, suppose that all CNP domains of depth $n-1$ satisfy NERD. Pick a CNP domain $\mathcal{P}$ on $X$ of depth $n$ with the associated factorization $q=\prod_{k=1}^{n} q_{k}$, which already determines the factors for Point 1 in the definition of NERD. By the definition of CNP domains one obtains $\mathcal{P}$ from a cyclical permutation domain on $X^{\prime}=\left\{X_{1}, \ldots, X_{q_{n}}\right\}$, where the sets of $X^{\prime}$ partition $X$ into $q_{n}$ equally sized sets. These $q_{n}$ sets deliver us the single indexed sets of the required nested set system. It follows from the replacement mechanism of the definition of CNP domains that any preference in $\mathcal{P}$ ranks either any alternative from $X_{i} \in X^{\prime}$ higher than any alternative from $X_{j} \in X^{\prime}$, or any alternative from $X_{i} \in X^{\prime}$ lower than any alternative from $X_{j} \in X^{\prime}$; this guarantees Point 2(a) of the NERD conditions. Moreover, each set $X_{i} \in X^{\prime}$ has to be replaced with a CNP domain on $X_{i}$ with the associated factorization $\prod_{k=1}^{n-1} q_{k}$ such that for fixed bijections $\varphi_{i, j}: X_{i} \rightarrow X_{j}$ we have that $x \in X_{i}$ and $\varphi_{i, j}(x)$ maintain their rank differences for all $x \in X_{i}$ whenever $x$ is ranked above $\varphi_{i, j}(x)$, which ensures Point 2(b) of the definition of NERD. By employing the induction hypothesis, NERD is satisfied by all CNP domains of depth $n-1$ on $X_{i} \in X^{\prime}$. Thus, there exist nested set systems

$$
\left\{X_{i}, X_{i_{n-1}, i}, \ldots, X_{i_{2}, \ldots, i_{n-1}, i}, X_{i_{1}, i_{2}, \ldots, i_{n-1}, i}\right\}_{i_{n-1}=1, \ldots, i_{1}=1}^{q_{n-1}, \ldots, q_{1}}
$$

on $X_{i}$ such that any CNP domain on $X_{i} \in X^{\prime}$ satisfies Points 1-3 of the definition of NERD for all $i=1, \ldots, q_{n}$. Taking the union of these $q_{n}$ nested set systems and $X$, we obtain the required nested set system for the domain $\mathcal{P}$. 
To prove the converse statement take a minimally rich domain $\mathcal{P}$ satisfying NERD. We show that $\mathcal{P}$ is a CNP domain by induction on $n$ in the definition of NERD. For $n=1$, NERD boils down to ERD, and therefore $\mathcal{P}$ is a cyclic permutation domain by Barbie, Puppe and Tasnádi (2006). Assume that a minimally rich domain $\mathcal{P}$ is a CNP domain if it satisfies NERD with $n-1$, and consider a minimally rich domain $\mathcal{P}$ satisfying NERD for a nested set system with $n$ indices. Point 1 in the definition of NERD delivers us the associated factorization of $\mathcal{P}$ and Point 2 determines the appropriate partition $X^{\prime}=\left\{X_{1}, \ldots, X_{q_{n}}\right\}$ of $X$. Define the domain $\mathcal{P}^{\prime} \subseteq \mathcal{P}_{X^{\prime}}$ by requiring for all $\succ^{\prime} \in \mathcal{P}^{\prime}$ that

$$
Y_{1} \succ^{\prime} \ldots \succ^{\prime} Y_{q_{n}} \Longleftrightarrow \exists \succ \in \mathcal{P}, \forall y_{1} \in Y_{1}, \ldots, \forall y_{q_{n}} \in Y_{q_{n}}, y_{1} \succ \ldots \succ y_{q_{n}}
$$

where $Y_{i} \in X^{\prime}$ for all $i=1, \ldots, q_{n}$ and $\cup_{i=1}^{q_{n}} Y_{i}=X$. Observe that if there exists $y_{1} \in Y_{1}, \ldots, y_{q_{n}} \in Y_{q_{n}}$ such that $y_{1} \succ \ldots \succ y_{q_{n}}$, then we must have $y_{1}^{\prime} \succ \ldots \succ y_{q_{n}}^{\prime}$ for all $y_{1}^{\prime} \in Y_{1}, \ldots, y_{q_{n}}^{\prime} \in Y_{q_{n}}$ by Point 2(a). Moreover, the non-emptyness of $\mathcal{P}$ implies the non-emptiness of $\mathcal{P}^{\prime}$. Since by Point 2(b) there exist bijections $\varphi_{i, j}^{n}: Y_{i} \rightarrow Y_{j}$ such that for all $y \in Y_{i}$ and all $\succ, \succ^{\prime} \in \mathcal{P}$ if $y \succ z=\varphi_{i, j}^{n}(y)$ and $y \succ^{\prime} z$, then

$$
d_{y, z}=r k[y, \succ]-r k[z, \succ]=r k\left[y, \succ^{\prime}\right]-r k\left[z, \succ^{\prime}\right]
$$

we must have by the definition of $\mathcal{P}^{\prime}$ that

$$
\frac{d_{y, z}}{q / q_{n}}=r k\left[Y_{i}, \succ^{*}\right]-r k\left[Y_{j}, \succ^{*}\right]=r k\left[Y_{i}, \succ^{* *}\right]-r k\left[Y_{j}, \succ^{* *}\right]
$$

whenever $Y_{i} \succ^{*} Y_{j}$ and $Y_{i} \succ^{* *} Y_{j}$, where $y \in Y_{i}$ and $z=\varphi_{i, j}^{n}(y)$. Hence, $\mathcal{P}^{\prime}$ satisfies ERD, and from the minimal richness condition it follows that $\mathcal{P}^{\prime} \subseteq \mathcal{P}_{X^{\prime}}$ has to be a cyclic permutation domain. Moreover, by Point 3 the distinct nested set systems

$$
\left\{X_{i}, X_{i_{n-1}, i}, \ldots, X_{i_{2}, \ldots, i_{n-1}, i}, X_{i_{1}, i_{2}, \ldots, i_{n-1}, i}\right\}_{i_{n-1}=1, \ldots, i_{1}=1}^{q_{n-1}, \ldots, q_{1}}
$$

satisfy NERD for all $i=1, \ldots, q_{n}$ with the same associated factorization $\prod_{k=1}^{n-1} q_{k}$. This means by the induction hypothesis that $\mathcal{P}_{i} \subseteq \mathcal{P}_{X_{i}}$ form CNP domains with associated factorizations $\prod_{k=1}^{n-1} q_{k}$ for all $i=1, \ldots, q_{n}$. Finally, Point 2 in the definition of NERD assures that $\mathcal{P}$ has to be obtained from the CNP domains on $X_{i}$ in line with the replacement mechanism specified in the definition of CNP domains, which completes the proof of Proposition 1.

Now we outline a procedure deciding whether a given domain $\mathcal{P}$ on $X$ is a $\mathrm{CNP}$ domain.

1. If $\# X \neq \# \mathcal{P}$, then $\mathcal{P}$ is not a CNP domain.

2. Pick a preference $\succ_{1} \in \mathcal{P}$ and label the alternatives so that $r k\left[x_{i}, \succ_{1}\right]=i$. Next, label the remaining preferences so that $r k\left[x_{1}, \succ_{i}\right]=i$. If this cannot be done, then $\mathcal{P}$ cannot be a CNP domain by Lemma 2.1 .

3. Step 3 aims to determine the associated factorization of $\mathcal{P}$. Let $r=\min \{i \in$ $\left.\{2, \ldots, q\} \mid r k\left[x_{i}, \succ_{2}\right]=1\right\}, n=1, q_{1}=r$.

(a) We have determined the associated factorization if $r=q$. 
(b) If $r$ is not a divisor of $q$, then $\mathcal{P}$ cannot be a CNP domain.

(c) If $r$ is a proper divisor of $q$, then let $s=\min \left\{i \in\{2 r, 3 r, \ldots, q\} \mid x_{i} \succ_{r+1} x_{1}\right\}$, increase $n$ by 1 , let $q_{n}=s / r, r=s$ and return to Substep (a).

4. Based on the factorization obtained in Step 3, we determine the nested set system by considering preference $\succ_{1}$ for which we have $x_{1} \succ_{1} x_{2} \succ \ldots \succ x_{q_{n}}$. First, let $q^{\prime}=q / q_{n}$ and let the single indexed sets be $X_{1}=\left\{x_{1}, x_{2}, \ldots, x_{q^{\prime}}\right\}$, $X_{2}=\left\{x_{q^{\prime}+1}, x_{q^{\prime}+2}, \ldots, x_{2 q^{\prime}}\right\}, \ldots, X_{q_{n}}=\left\{x_{q-q^{\prime}+1}, x_{q-q^{\prime}+2}, \ldots, x_{q}\right\}$. To determine the double indexed sets we have to partition the single indexed sets by taking consecutive sequences of length $q /\left(q_{n-1} q_{n}\right)$ from the sequence $x_{1}, x_{2}, \ldots, x_{q}$. We have to proceed in a similar way to obtain the remaining sets. More formally, for all $k=0, \ldots, n-1$ let $q_{k}^{*}=\prod_{l=1}^{k} q_{l}$ and for all $k=1, \ldots, n$, all $\left(i_{k}, \ldots, i_{n}\right) \in \times_{l=k}^{n}\left\{1, \ldots, q_{l}\right\}$ let $j=\left(\sum_{l=k}^{n}\left(i_{l}-1\right) q_{l-1}^{*}\right)+1$ and $X_{i_{k}, i_{k+1}, \ldots, i_{n}}=$ $\left\{x_{j}, x_{j+1}, \ldots, x_{j+q_{k-1}^{*}-1}\right\}$.

5. It is straightforward to check whether the NERD conditions 2(a) and 3(b) are satisfied.

6. To find appropriate bijections $\varphi_{i, j}^{l-1}: X_{i, i_{l}, \ldots, i_{n-1}, i_{n}} \rightarrow X_{j, i_{l}, \ldots, i_{n-1}, i_{n}}$ that satisfy NERD conditions $2(\mathrm{~b})$ and $3(\mathrm{c})$ pick for each $x \in X_{i, i_{l}, \ldots, i_{n-1}, i_{n}}$ the preference $\succ \in \mathcal{P}$ ranking $x$ on top, then the highest ranked alternative $y$ out of $X_{j, i_{l}, \ldots, i_{n-1}, i_{n}}$ by $\succ$, and let $\varphi_{i, j}^{l-1}(x)=y$. If this cannot be done, $\mathcal{P}$ fails to be a minimally rich domain, and thus, to be a CNP domain. If $\varphi_{i, j}^{l-1}$ is not a bijection, then $\mathcal{P}$ cannot be a CNP domain. Otherwise, verify NERD conditions 2(b) and 3(c).

Clearly, only CNP domains are accepted by the above procedure. However, that any CNP domain is accepted by the procedure is less obvious. CNP domains pass Steps 1 and 2 by Lemma 2.1. Assume that we have labeled the preferences of $\mathcal{P}$ according to Step 2. We verify by induction on $n$ in the definition of NERD that $\mathcal{P}$ passes Steps 3-6. If $n=1$, then $\mathcal{P}$ is a cyclic permutation domain, and thus, $r=q$ by Step 3. Moreover, Step 4 determines the nested set system $\left\{X,\left\{x_{1}\right\}, \ldots,\left\{x_{q}\right\}\right\}$ associated with cyclic permutation domains. Since $\mathcal{P}$ satisfies NERD, it also passes Steps 5 and 6. Assume that the procedure works well for nested set systems with $n-1$ factors and take a domain $\mathcal{P}$ needing $n$ factors. Let $X^{\prime}=\left\{X_{1}, \ldots, X_{q_{n}}\right\}$ be the set of the single labeled sets from the given nested set system and assume that the sets are labeled in a way such that $X_{1}=\left\{x_{1}, \ldots, x_{q / q_{n}}\right\}, \ldots, X_{q_{n}}=\left\{x_{q-q / q_{n}+1}, \ldots, x_{q}\right\}$. Define $\succ^{\prime} \in \mathcal{P}_{X^{\prime}}$ by

$$
X_{1} \succ^{\prime} X_{2} \succ^{\prime} \ldots \succ^{\prime} X_{q_{n}} \Longleftrightarrow x_{1} \succ_{1} x_{q / q_{n}+1} \succ_{1} \ldots \succ_{1} x_{q-q / q_{n}+1},
$$

which is well defined by Point 2 of NERD. Moreover, by Point 2 of NERD we obtain $\mathcal{P}$ from $\mathcal{Z}\left(\succ^{\prime}\right)$, and therefore, only the alternatives from $X_{q_{n}}$ are ranked higher than $x_{1}$ by $\succ_{q / q_{n}+1}$. Hence, Step 3 determines the last factor $q_{n}$ correctly. Finally, the induction hypothesis assures that Steps 3-6 work correctly.

\section{Proper Scoring Methods on CNP Domains}

Definition (Scoring method) Let $q$ be the cardinality of $X$, and let $s:\{1, \ldots, q\} \rightarrow \mathbf{R}$ satisfy $s(1) \geq s(2) \geq \ldots \geq s(q)$ and $s(1)>s(q)$. The SCR $f^{s}$ associated with the scoring 
method specified by $s$ is given by

$$
x \in f^{s}\left(\succ_{1}, \ldots, \succ_{n}\right) \Leftrightarrow \sum_{i=1}^{n} s\left(r k\left[x, \succ_{i}\right]\right) \geq \sum_{i=1}^{n} s\left(r k\left[y, \succ_{i}\right]\right) \quad \text { for all } y \in X
$$

for all $n$ and all $\succ_{1}, \ldots, \succ_{n} \in \mathcal{P}_{X}$. A scoring method is called proper if $s$ is strictly decreasing.

Definition (Nested Borda count) Assume that there are $n$ given integers $q_{k} \geq 2$ for all $k=1, \ldots, n$ such that $q=\prod_{k=1}^{n} q_{k}$ and $n$ positive reals $\delta_{1}, \ldots, \delta_{n}$ such that $\delta_{i+1} \geq \delta_{i} q_{i}$ for all $i=1, \ldots, n-1$. Let $q_{k}^{*}=\prod_{i=1}^{k} q_{i}$ for all $k=0, \ldots, n-1$; and let $j_{i_{k}, \ldots, i_{n}}=\left(\sum_{l=k}^{n}\left(i_{l}-1\right) q_{l-1}^{*}\right)+1$ for all $k=1, \ldots, n$, all $\left(i_{k}, \ldots, i_{n}\right) \in \times_{l=k}^{n}\left\{1, \ldots, q_{l}\right\}$. We call a proper scoring method a Nested Borda count with the associated factors $q_{1}, \ldots, q_{n}$ if the score function $s$ satisfies $s\left(j_{i, i_{k+1}, \ldots, i_{n}}\right)-s\left(j_{i+1, i_{k+1}, \ldots, i_{n}}\right)=\delta_{k}$ for all $i=1, \ldots, q_{k}-1$, all $\left(i_{k+1}, \ldots, i_{n}\right) \in \times_{l=k+1}^{n}\left\{1, \ldots, q_{l}\right\}$ and all $k=1, \ldots, n$.

It can be verified that a Nested Borda count boils down to the Borda count if $\delta_{i+1}=\delta_{i} q_{i}$ for all $i=1, \ldots, n-1$ or if $n=1$. In particular, for any factorization $q=\prod_{k=1}^{n} q_{k}$ we obtain the score function of the Borda count if $\delta_{1}=1$ and $\delta_{i+1}=\delta_{i} q_{i}$ for all $i=1, \ldots, n-1$. Informally, the Borda count on $q$ alternatives can be obtained through all factorizations of $q$.

If one considers the nested set system consisting of sets

$$
X_{i_{k}, i_{k+1}, \ldots, i_{n}}=\left\{j_{i_{k}, i_{k+1}, \ldots, i_{n}}, \ldots, j_{i_{k}, i_{k+1}, \ldots, i_{n}}+q_{k-1}^{*}-1\right\}
$$

for all $\left(i_{k}, \ldots, i_{n}\right) \in \times_{l=k}^{n}\left\{1, \ldots, q_{l}\right\}$ and all $k=1, \ldots, n$, then any positive integer $j=1, \ldots, q$ uniquely specifies indices $i_{1}, i_{2}, \ldots, i_{n}$ such that $j \in X_{i_{k}, i_{k+1}, \ldots, i_{n}}$ for all $k=1, \ldots, n$. Taking for all $k=1, \ldots, n$ the Borda score functions $s_{k}(i)=q_{k}+1-i$ on $q_{k}$ alternatives, we can obtain the score function $s$ of a Nested Borda count by $s(j)=\left(\sum_{k=1}^{n} \delta_{k}\left(s_{k}\left(i_{k}\right)-1\right)\right)+\alpha$, where $\alpha \in \mathbf{R}$. This is the reason why we refer to the above defined class of scoring methods as Nested Borda counts.

Proposition 2 Let $\mathcal{P}$ be a CNP domain with associated factorization $q=\prod_{k=1}^{n} q_{k}$. A proper scoring method is monotonic on $\mathcal{P}$ if and only if it is a Nested Borda count with associated factors $q_{1}, \ldots, q_{n}$.

Proof Since $\mathcal{P}$ is a CNP domain, $\mathcal{P}$ satisfies NERD. Thus, we can consider the corresponding nested set system

$$
\left\{X, X_{i_{n}}, X_{i_{n-1}, i_{n}}, \ldots, X_{i_{2}, \ldots, i_{n-1}, i_{n}}, X_{i_{1}, i_{2}, \ldots, i_{n-1}, i_{n}}\right\}_{i_{n}=1, \ldots, i_{1}=1}^{q_{n}, \ldots, q_{1}}
$$

and let $q_{k}^{*}=\prod_{i=1}^{k} q_{i}$ for all $k=0, \ldots, n-1$.

Suppose that the proper scoring method $s$ is not a Nested Borda count, and therefore, there exists a smallest $k \in\{1, \ldots, n\}$ such that

$$
d_{1}=s\left(j_{i+1, i_{k+1}, \ldots, i_{n}}\right)-s\left(j_{i, i_{k+1}, \ldots, i_{n}}\right) \neq d_{2}=s\left(j_{i+2, i_{k+1}, \ldots, i_{n}}\right)-s\left(j_{i+1, i_{k+1}, \ldots, i_{n}}\right)
$$

for some $i \in\left\{1, \ldots, q_{k}-2\right\}$ and $\left(i_{k+1}, \ldots, i_{n}\right) \in \times_{l=k+1}^{n}\left\{1, \ldots, q_{l}\right\}$. Take a profile $\Pi$ containing exactly one voter of each type, for instance, let $\Pi=\left(\succ_{1}, \ldots, \succ_{q}\right)$. Hence, $f^{s}(\Pi)=X$ by Lemma 2.1. By the NERD conditions we can pick two distinct sets 
$X_{j, i_{k+1}, \ldots, i_{n-1}, i_{n}}$ and $X_{j^{\prime}, i_{k+1}, \ldots, i_{n-1}, i_{n}}$ for which there exist two sets of preferences $\mathcal{P}_{1}, \mathcal{P}_{2} \subseteq \mathcal{P}$ such that $\# \mathcal{P}_{1}=\# \mathcal{P}_{2}=q_{k-1}^{*}$,

$$
\begin{aligned}
& j_{i, i_{k+1}, \ldots, i_{n}} \leq r k[x, \succ]<j_{i+1, i_{k+1}, \ldots, i_{n}}, \\
& j_{i+1, i_{k+1}, \ldots, i_{n}} \leq r k[y, \succ]<j_{i+2, i_{k+1}, \ldots, i_{n}}, \\
& j_{i+1, i_{k+1}, \ldots, i_{n}} \leq r k\left[x, \succ^{\prime}\right]<j_{i+2, i_{k+1}, \ldots, i_{n}}, \\
& j_{i+2, i_{k+1}, \ldots, i_{n}} \leq r k\left[y, \succ^{\prime}\right]<j_{i+2, i_{k+1}, \ldots, i_{n}}+q_{k-1}^{*}
\end{aligned}
$$

for all $x \in X_{j, i_{k+1}, \ldots, i_{n-1}, i_{n}}$, all $y \in X_{j^{\prime}, i_{k+1}, \ldots, i_{n-1}, i_{n}}$, all $\succ \in \mathcal{P}_{1}$ and all $\succ^{\prime} \in \mathcal{P}_{2}$. We construct profile $\Pi^{\prime}$ from profile $\Pi$ by replacing those voters' preferences having preferences in $\mathcal{P}_{2}$ by preferences in $\mathcal{P}_{1}$ appropriately. More specifically, if $\succ \in \mathcal{P}_{2}$, then $\succ$ has to replaced with the preference $\succ^{\prime} \in \mathcal{P}_{1}$ satisfying

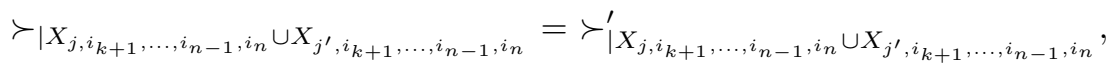

which can be done by the NERD condition. Moreover, the NERD condition guarantees that profiles $\Pi$ and $\Pi^{\prime}$ satisfy the precondition of monotonicity at any alternative $X_{j, i_{k+1}, \ldots, i_{n-1}, i_{n}} \cup X_{j^{\prime}, i_{k+1}, \ldots, i_{n-1}, i_{n}} \subseteq f^{s}(\Pi)$. It can be verified that $X_{j, i_{k+1}, \ldots, i_{n-1}, i_{n}} \cap$ $f^{s}(\Pi)=\emptyset$ if $d_{1}<d_{2}$, while $X_{j^{\prime}, i_{k+1}, \ldots, i_{n-1}, i_{n}} \cap f^{s}\left(\Pi^{\prime}\right)=\emptyset$ if $d_{1}>d_{2}$. Hence, monotonicity is violated either at any alternative in $X_{j, i_{k+1}, \ldots, i_{n-1}, i_{n}}$ or any alternative in $X_{j^{\prime}, i_{k+1}, \ldots, i_{n-1}, i_{n}}$.

Now we verify that a Nested Borda count $f^{s}$ with the associated factorization $q=\prod_{k=1}^{n} q_{k}$ is monotonic on a CNP domain $\mathcal{P}$ with the same associated factorization. We employ an induction on $n$. If $n=1$, then $\mathcal{P}$ is a simple cyclic permutation domain and $f^{s}=f^{B}$. Pick an arbitrary profile $\Pi$ and any alternative $x \in f(\Pi)$. Note that for any cyclic permutation domain $L(x, \succ) \subseteq L\left(x, \succ^{\prime}\right)$ implies for any other alternative $y \in X \backslash\{x\}$ either equal rank differences in $\succ$ and $\succ^{\prime}$ between $x$ and $y$ or $y \succ x$ and $x \succ^{\prime} y$. Thus, $x$ cannot be overtaken by other alternatives if we replace preferences in $\Pi$ with other preferences in a way that the precondition of monotonicity is satisfied. Thus, a cyclic permutation domain has to be Borda monotonic.

Assume that any Nested Borda count with $n-1$ factors is monotonic on a CNP domain of depth $n-1$ with identical factorization. Take an arbitrary Nested Borda count $f^{s}$ with the associated factorization $q=\prod_{k=1}^{n} q_{k}$ and arbitrary CNP domain $\mathcal{P}$ with the same associated factorization. We shall denote by $X^{\prime}=\left\{X_{1}, \ldots, X_{q_{n}}\right\}$ the partition required in the definition of CNP domains. By the construction of CNP domains there are for all $i, j \in\left\{1, \ldots, q_{n}\right\}$ and $i \neq j$ bijections $\varphi_{i, j}: X_{i} \rightarrow X_{j}$ such that $x \in X_{i}$ and $\varphi_{i, j}(x)$ maintain their rank differences whenever $x$ is ranked above $\varphi_{i, j}(x)$. Since the replacement mechanism assures that any alternative $X_{i}\left(i=1, \ldots, q_{n}\right)$ has to be replaced by a CNP domain on $X_{i}$ with the associated factorization $\prod_{k=1}^{n-1} q_{k}$ and the restriction $f^{s^{\prime}}$ of $f^{s}$ to $q / q_{n}$ alternatives (i.e., $s^{\prime}:\left\{1, \ldots, q / q_{n}\right\} \rightarrow \mathbf{R}$ such that $s^{\prime}(i)=s(i)$ for all $\left.i=1, \ldots, q / q_{n}\right)$ with the associated factorization $\prod_{k=1}^{n-1} q_{k}$ is monotonic on $\mathcal{P}_{\mid X_{i}}$ by our induction hypothesis, we can only have a violation of monotonicity by considering two alternatives belonging to two distinct sets $X_{i}$ and $X_{j}$. Take an arbitrary profile $\Pi$ such that $x \in f(\Pi)$, where $x \in X_{i}$. Alternative $x$ can be overtaken by alternative $y \in X_{j}(i \neq j)$ by replacing preferences in $\Pi$ without violating the precondition of monotonicity only if we can find voters of type $\succ$ in $\Pi$ and a preference $\succ^{\prime} \in \mathcal{P}$ such that $L(x, \succ) \subseteq L\left(x, \succ^{\prime}\right)$ and either

(a) $x \succ y, x \succ^{\prime} y$ and $s(r k[x, \succ])-s(r k[y, \succ])>s\left(r k\left[x, \succ^{\prime}\right]\right)-s\left(r k\left[y, \succ^{\prime}\right]\right)$ or 
(b) $y \succ x, y \succ^{\prime} x$ and $s(r k[y, \succ])-s(r k[x, \succ])<s\left(r k\left[y, \succ^{\prime}\right]\right)-s\left(r k\left[x, \succ^{\prime}\right]\right)$.

We only consider case (a) since case (b) can be established in an analogous way. Let $x^{\prime}=\varphi_{i, j}^{-1}(y), d=s\left(r k\left[x^{\prime}, \succ\right]\right)-s(r k[y, \succ])=s\left(r k\left[x^{\prime}, \succ^{\prime}\right]\right)-s\left(r k\left[y, \succ^{\prime}\right]\right), d_{1}=$ $s(r k[x, \succ])-s(r k[y, \succ])$ and $d_{2}=s\left(r k\left[x, \succ^{\prime}\right]\right)-s\left(r k\left[y, \succ^{\prime}\right]\right)$. We cannot have $x \succ x^{\prime}$ and $x^{\prime} \succ^{\prime} x$, since this would violate $L(x, \succ) \subseteq L\left(x, \succ^{\prime}\right)$. Moreover, $x^{\prime} \succ x$ and $x \succ^{\prime} x^{\prime}$ cannot be the case, since this would imply $d_{1}<d<d_{2}$, which is in contradiction with $d_{1}>d_{2}$. The remaining two subcases $x \succ x^{\prime}$ and $x \succ^{\prime} x^{\prime}$, and $x^{\prime} \succ x$ and $x^{\prime} \succ^{\prime} x$ would imply the non-monotonicity of $f^{s^{\prime}}$ on a CNP domain of depth $n-1$ and identical factorization as $s^{\prime}$ on $X_{i}$; a contradiction; which completes the proof of our proposition.

Corollary 2 Within the class of proper scoring methods only the Borda count can be monotonic on all CNP domains.

\section{Some Useful Lemmas}

For the proof of the theorem, we need a series of lemmas some of which are interesting on their own right. If there are $k$ given preferences $\succ_{1}, \ldots, \succ_{k} \in \mathcal{P}$ and $k$ given positive integers $n_{1}, \ldots, n_{k}$, then we shall denote by $\Pi=\left(n_{1} \cdot \succ_{1}, \ldots, n_{k} \cdot \succ_{k}\right)$ a preference profile in which the first $n_{1}$ voters have preference $\succ_{1}$, the next $n_{2}$ voters have preference $\succ_{2}$ and so on.

Lemma 5.1 Suppose that $\mathcal{P}$ is a minimally rich domain. If there exist two distinct preferences $\succ, \succ^{\prime} \in \mathcal{P}$ and two alternatives $x, y \in X$ satisfying $r k[x, \succ]=1, r k[y, \succ]=$ 2 and $d:=r k\left[y, \succ^{\prime}\right]-r k\left[x, \succ^{\prime}\right] \geq 2$, then Borda count violates monotonicity on $\mathcal{P}$.

Proof of Lemma 5.1 Let $\succ^{\prime \prime} \in \mathcal{P}$ be a preference with top alternative $y, d^{\prime}=$ $r k\left[x, \succ^{\prime \prime}\right]-r k\left[y, \succ^{\prime \prime}\right]$ and $k=\left[\frac{2 q+1}{d^{\prime}+1}\right] \cdot{ }^{8}$ We consider the following two profiles of $k\left(d^{\prime}+1\right)-1$ individuals: $\Pi=\left(\left(k d^{\prime}-1\right) \cdot \succ, k \cdot \succ^{\prime \prime}\right)$ and $\Pi^{\prime}=\left(\left(k d^{\prime}-3\right) \cdot \succ, 2 \cdot \succ^{\prime}, k \cdot \succ^{\prime \prime}\right)$. Observe that the Borda score of $y$ is greater than that of $x$ by 1 in profile $\Pi$ and since $y$ dominates the remaining alternatives we have $f^{B}(\Pi)=\{y\}$. For profile $\Pi^{\prime}$ the choice of $k$ assures ${ }^{9}$ that $x$ and $y$ receive higher Borda scores than any other alternative. Thus, $f^{B}\left(\Pi^{\prime}\right)=\{x\}$ by the assumptions imposed on $\succ$ and $\succ^{\prime}$. Finally, the precondition of monotonicity for the alternative $x$ is satisfied as we switch from $\Pi^{\prime}$ to $\Pi$, but $y$ becomes the Borda winning alternative in $\Pi$. This completes the proof.

Lemma 5.2 If $\mathcal{P}$ is a Borda monotonic minimally rich domain, then for any two preferences in $\mathcal{P}$ having the same top alternative the second ranked alternatives have to be identical.

Proof of Lemma 5.2 Suppose there are preferences $\succ, \succ^{\prime} \in \mathcal{P}$ such that $r k[x, \succ]=1$, $r k[y, \succ]=2, r k\left[x, \succ^{\prime}\right]=1, r k\left[z, \succ^{\prime}\right]=2$ and $y \neq z$. Then $r k\left[y, \succ^{\prime}\right]>2$ and Lemma 5.1 applies.

\footnotetext{
${ }^{8}$ In what follows $\lfloor x\rfloor$ stands for the largest integer not greater than $x$ and $\lceil x\rceil$ stands for the smallest integer not less than $x$.

${ }^{9}$ Any larger integer for $k$ does the job.
} 
Lemma 5.3 If $\mathcal{P}$ is a Borda monotonic minimally rich domain, then

$$
\pi(x)=\{y \in X \mid \exists \succ \in \mathcal{P} \text { such that } r k[x, \succ]=1 \text { and } r k[y, \succ]=2\}
$$

defines a one-to-one correspondence (permutation) on $X$.

Proof of Lemma 5.3 The statement is obviously true in case of $q \leq 3$. Therefore, we only have to consider the case of $q>3$. Suppose that $x$ is ranked first by $\succ \in \mathcal{P}$ and ranked second by $\succ^{\prime}, \succ^{\prime \prime} \in \mathcal{P}$. We shall denote the top alternatives of $\succ^{\prime} \in \mathcal{P}$ and $\succ^{\prime \prime} \in \mathcal{P}$ by $y$ and $z$, respectively. Any $\succ^{*} \in \mathcal{P} \backslash\left\{\succ, \succ^{\prime}, \succ^{\prime \prime}\right\}$ has to rank $y$ or $z$ lower than $x$; since otherwise, $y$ and $x$ or $z$ and $x$ violate Lemma 5.1. Hence, $\mathcal{P}$ cannot satisfy part (ii) of the minimal richness condition, a contradiction.

Lemma 5.4 Suppose that $\mathcal{P}$ is a Borda monotonic minimally rich domain. Then we cannot find two distinct preferences $\succ, \succ^{\prime} \in \mathcal{P}$ and an alternative $y \in X$ such that

- $r k[y, \succ]>2$

- $U(y, \succ)=U\left(y, \succ^{\prime}\right)$,

- $\forall x \in U(y, \succ): r k[x, \succ] \neq r k\left[x, \succ^{\prime}\right]$.

Proof of Lemma 5.4 Suppose that there exist two distinct preferences $\succ, \succ^{\prime} \in \mathcal{P}$ and an alternative $y \in X$ such that $d=r k[y, \succ]>2, U(y, \succ)=U\left(y, \succ^{\prime}\right)$ and $r k[x, \succ] \neq$ $r k\left[x, \succ^{\prime}\right]$ for all $x \in U(y, \succ)$. Let $\succ^{\prime \prime} \in \mathcal{P}$ be a preference with top alternative $y$ and $U(y, \succ)=\left\{x_{1}, \ldots, x_{d-1}\right\}$. Observe that $y$ dominates all alternatives in $X \backslash U(y, \succ)$ in all profiles consisting only of preferences $\succ, \succ^{\prime}$ and $\succ^{\prime \prime}$. We define values $d_{m}=$ $2 d-r k\left[x_{m}, \succ\right]-r k\left[x_{m}, \succ^{\prime}\right]$ and $d_{m}^{\prime}=r k\left[x_{m}, \succ^{\prime \prime}\right]-1$ for all $m \in\{1, \ldots, d-1\}$. Now let $S=\arg \min _{s \in\{1, \ldots, d-1\}} \frac{d_{s}^{\prime}}{d_{s}}$ and $A=\left\{x_{s} \in X \mid s \in S\right\}$. For any $s \in S$ it can be verified that a profile consisting of $d_{s}^{\prime}$ preferences of type $\succ, d_{s}^{\prime}$ preferences of type $\succ^{\prime}$ and $d_{s}$ preferences of type $\succ^{\prime \prime}$ makes alternatives $\{y\} \cup A$ indifferent on the top. Let $\Pi=\left(d_{s}^{\prime} \cdot \succ, d_{s}^{\prime} \cdot \succ^{\prime}, d_{s} \cdot \succ^{\prime \prime}\right)$. Hence, $f^{B}(\Pi)=\{y\} \cup A$.

First, if there exists an $s \in S$ such that $x_{s}$ is ranked higher in $\succ^{\prime}$ than in $\succ$, then pick an arbitrary alternative $x_{m} \in A$ achieving the highest rank increase by replacing one voter of type $\succ$ with one voter of type $\succ^{\prime}$. In this case we construct $\Pi^{\prime}$ from $\Pi$ by replacing one preference $\succ$ with one preference $\succ^{\prime}$. It can be checked that $y \notin f^{B}\left(\Pi^{\prime}\right)$, while $x_{m} \in f^{B}\left(\Pi^{\prime}\right)$. Second, if for all $s \in S$ we have that $x_{s}$ is ranked higher in $\succ$ than in $\succ^{\prime}$, then pick an arbitrary alternative $x_{m} \in A$ achieving the highest rank decrease from $\succ$ to $\succ^{\prime}$. In this second case we construct $\Pi^{\prime}$ from $\Pi$ by replacing one preference $\succ^{\prime}$ with one preference $\succ$. Again, we have $y \notin f^{B}\left(\Pi^{\prime}\right)$, while $x_{m} \in f^{B}\left(\Pi^{\prime}\right)$. We obtained in both cases a violation of monotonicity at $y$; a contradiction.

Lemma 5.5 Let $\mathcal{P}$ be a Borda monotonic minimally rich domain, $X^{\prime} \subseteq X$ and $\mathcal{P}^{\prime} \subseteq$ $\mathcal{P}$. Assume that $q^{\prime}:=\# X^{\prime}=\# \mathcal{P}^{\prime}$ and that the restriction of $\mathcal{P}^{\prime}$ to its top $q^{\prime}$ alternatives gives a CNP domain on $X^{\prime}$. Then for any preference $\succ \in \mathcal{P}$ there exists a preference $\succ^{\prime} \in \mathcal{P}^{\prime}$ such that the alternatives from $X^{\prime}$ must follow each other consecutively in the same order in $\succ$ as in $\succ^{\prime}$.

Proof of Lemma 5.5 The restriction of $\mathcal{P}^{\prime}$ to its top $q^{\prime}$ alternatives equals $\mathcal{P}_{\mid X^{\prime}}^{\prime}$, which is a CNP domain on $X^{\prime}$, by the assumptions of Lemma 5.5. We employ an induction 
on the depth of the CNP domain on $X^{\prime}$. Lemma 5.1 implies that Lemma 5.5 is satisfied whenever $\mathcal{P}_{\mid X^{\prime}}^{\prime}$ is a CNP domain of depth 1 .

Assume that the statement holds true for any CNP domain $\mathcal{P}_{\mid X^{\prime}}^{\prime}$ of depth less than $n$. Now let $\mathcal{P}_{\mid X^{\prime}}^{\prime}$ be a CNP domain of depth $n$ and take the partition of $X^{\prime}$ into sets $X_{1}, \ldots, X_{k}$ of cardinality $q^{\prime} / k$ according to the definition of CNP domains. Then $\mathcal{P}_{\mid X_{i}}^{\prime}$ are CNP domains of depth $n-1$ for all $i=1, \ldots, k$. Thus, for all preferences $\succ \in \mathcal{P}$ and all $i=1, \ldots, k$ there exists a preference $\succ^{\prime} \in \mathcal{P}^{\prime}$ such that the alternatives from $X_{i}$ must follow each other consecutively in the same order in $\succ$ as in $\succ^{\prime}$ by our induction hypothesis. Pick an arbitrary preference $\succ \in \mathcal{P} \backslash \mathcal{P}^{\prime}$ and suppose that there does not exist a preference $\succ^{\prime} \in \mathcal{P}^{\prime}$ such that the alternatives from $X^{\prime}$ must follow each other consecutively in the same order in $\succ$ as in $\succ^{\prime}$. Let $x_{1}$ be the highest ranked $X^{\prime}$ alternative by $\succ$. We can assume without loss of generality that $x_{1} \in$ $X_{1}$. We shall denote by $\succ^{\prime} \in \mathcal{P}^{\prime}$ the preference ranking $x_{1}$ on top. We assume for notational convenience that $\succ^{\prime} \operatorname{ranks} X_{i}$ above $X_{i+1}$ for all $i=1, \ldots, k-1$. Let $j \in\{1, \ldots, k\}$ be the largest index such that the alternatives $\cup_{i=1}^{j-1} X_{i}$ follow each other consecutively in the same order in $\succ$ as in $\succ^{\prime}$. We shall denote by $x_{j}$ the highest ranked $X_{j}$ alternative in $\succ^{\prime}$ and by $\succ^{\prime \prime} \in \mathcal{P}^{\prime}$ the preference with top alternative $x_{j}$. There exists positive integers $a$ and $b$ such that profile $\Pi=\left(a \cdot \succ^{\prime}, b \cdot \succ^{\prime \prime}\right)$ has $x_{j}$ and $U \subseteq \cup_{i=1}^{j-1} X_{i}$ indifferent on the top. We shall denote by $u$ the lowest ranked alternative from $U$ by $\succ^{\prime}$. Let $d^{\prime}=r k\left[x_{j}, \succ^{\prime}\right]-r k\left[u, \succ^{\prime}\right]$ and $d=r k\left[x_{j}, \succ\right]-r k[u, \succ]$. We must have $d^{\prime}<d$ by the definition of $j$ and our induction hypothesis. Let $c=\left[\frac{d^{\prime}}{d-d^{\prime}}\right]$. We can assume that $a>c$, since otherwise, we can take an appropriate multiple of $a$ and $b$ to have $f^{B}(\Pi)=\left\{x_{j}\right\} \cup U$ and $a>c$. Let $\Pi^{\prime}=\left((a-1) \cdot \succ^{\prime}, b \cdot \succ^{\prime \prime}\right)$ and $\Pi^{\prime \prime}=\left(c \cdot \succ,(a-1-c) \cdot \succ^{\prime}, b \cdot \succ^{\prime \prime}\right)$. If $a$ and $b$ were chosen large enough so that no other alternative can interfere, then $f^{B}\left(\Pi^{\prime}\right)=\left\{x_{j}\right\}$ and $u \in f^{B}\left(\Pi^{\prime \prime}\right)$, and therefore, monotonicity is violated at $u$ by switching from $\Pi^{\prime \prime}$ to $\Pi^{\prime}$.

\section{Proof of the Main Result}

Proof of Theorem Sufficiency follows from Proposition 2, since the Borda count is a Nested Borda count for any factorization of $q$.

Thus, we just have to prove the necessity of CNP domains for which we need the following notations. For any $1 \leq i \leq j \leq q=\# X$ let $\succ_{\mid[i, j]}$ be the restriction of $\succ$ ranging from the $i$ th position to the $j$ th position of $\succ$, i.e., $\succ_{\mid[i, j]}=\succ_{\mid\left\{x_{i}, x_{i+1}, \ldots, x_{j}\right\}}$ where $x_{1} \succ \cdots \succ x_{i} \succ \cdots \succ x_{j} \succ \cdots \succ x_{q}$. In addition, for any $1 \leq i \leq j \leq q$, we define $\mathcal{P}_{[i, j]}=\left\{\succ_{\mid[i, j]} \mid \succ \in \mathcal{P}\right\}$. Furthermore, for any linear ordering $\succ$ on $X^{\prime} \subseteq X$ we define $\left.T_{i}(\succ)=\left\{x \in X^{\prime} \mid r k[x, \succ] \leq i\right\}\right)$ and $M_{i, j}(\succ)=\left\{x \in X^{\prime} \mid i \leq r k[x, \succ] \leq j\right\}$. We divide our proof into several steps.

Step 1: We show that $\mathcal{P}$ can be partitioned into subdomains having cyclic permutation domains on top.

Lemma 5.3 implies that the top two alternatives determine a permutation $\pi$ of $X$. The cycles of permutation $\pi$ partition $X$ into sets $X_{1}, \ldots, X_{p}$. We shall denote by $X^{\prime}$ an arbitrary set $X_{i}(i=1, \ldots, p)$, by $x_{1}, \ldots, x_{m}$ its alternatives and by $\succ_{k} \in \mathcal{P}$ an arbitrary preference with top alternative $x_{k}(k=1, \ldots, m) .{ }^{10}$ Clearly, $m \geq 2$. Let

\footnotetext{
${ }^{10}$ It will turn out that the preference having $x_{k}$ on top is unique.
} 
$\mathcal{P}^{\prime}=\left\{\succ_{1}, \ldots, \succ_{m}\right\}$. In what follows we can assume without loss of generality that $\operatorname{rk}\left[x_{k \oplus_{m} 1}, \succ_{k}\right]=2 .{ }^{11}$

We determine the top $m$ alternatives of $\mathcal{P}^{\prime}$. We must have $r k\left[x_{k \oplus_{m} 2}, \succ_{k}\right]=3$ for all $k=1, \ldots, m$ by Lemma 5.1. Moreover, it follows from Lemma 5.1 by induction that $r k\left[x_{k \oplus_{m} l}, \succ_{k}\right]=l+1$ for all $l=1, \ldots, m-1$ and all $k=1, \ldots, m$. But this implies that the top $m$ alternatives of the preferences in $\mathcal{P}^{\prime}$ follow the pattern shown in Table 9. Moreover, the restriction to its top $m$ alternatives of any preference in $\mathcal{P}$ with a

Table 9: A full cycle on the top

\begin{tabular}{lllll}
$\succ_{1}$ & $\succ_{2}$ & $\ldots$ & $\succ_{m-1}$ & $\succ_{m}$ \\
\hline$x_{1}$ & $x_{2}$ & $\ldots$ & $x_{m-1}$ & $x_{m}$ \\
$x_{2}$ & $x_{3}$ & $\ldots$ & $x_{m}$ & $x_{1}$ \\
$\vdots$ & $\vdots$ &. & $\vdots$ & $\vdots$ \\
& &. & & \\
$x_{m-1}$ & $x_{m}$ & $\ldots$ & $x_{m-3}$ & $x_{m-2}$ \\
$x_{m}$ & $x_{1}$ & $\ldots$ & $x_{m-2}$ & $x_{m-1}$ \\
$\vdots$ & $\vdots$ & & $\vdots$ & $\vdots$
\end{tabular}

top alternative from $X^{\prime}$ equals the restriction to its top $m$ alternatives of a preference in $\mathcal{P}^{\prime}$. In addition, $\mathcal{P}^{\prime}$ prescribes the possible orderings of the alternatives from $X^{\prime}$ by any preference in $\mathcal{P}$ by Lemma 5.5 .

Clearly, we are finished if $p=1$. Hence, in what follows we will assume that $p>1$.

Step 2: We show that if $\mathcal{P}$ can be partitioned into subdomains having CNP domains on top, then a union of some of these subdomains has a deeper CNP domain on top.

Let $X_{1}, \ldots, X_{p}$ be a partition of $X, m_{i}=\# X_{i}$ and

$$
\mathcal{P}^{i}=\left\{\succ \in \mathcal{P} \mid \exists x \in X_{i} \text { such that } r k[x, \succ]=1\right\}
$$

for all $i=1, \ldots, p$. Assume that we have already established that $T_{m_{i}}(\succ)=X_{i}$ for all $\succ \in \mathcal{P}^{i}$ and that $\mathcal{P}_{\mid X_{i}}$ are CNP domains on $X_{i}$ for all $i=1, \ldots, p$.

We will demonstrate in Step 2 that Borda monotonicity implies the existence of a set of indices $I \subseteq\{1, \ldots, p\}$ such that $\# I \geq 2$ and $\mathcal{P}_{\mid Y}^{\prime}$ is a CNP domain on $Y$, where $Y=\cup_{i \in I} X_{i}, \mathcal{P}^{\prime}=\cup_{i \in I} \mathcal{P}^{i}$ and $T_{\# Y}(\succ)=Y$ for all $\succ \in \mathcal{P}^{\prime} .{ }^{12}$

We can assume without loss of generality that $m_{1} \leq m_{i}$ for all $i=1, \ldots, p$ and we simply write $m$ for $m_{1}$. Our proof of Step 2 will require three substeps.

Substep A: We show that the restriction of a subdomain of $\mathcal{P}$ with a CNP domain of size $m$ on top contains from its $m+1$ th to $2 m$ th positions another CNP domain of the same structure. Moreover, this latter CNP domain is on top of another subdomain of $\mathcal{P}$. This "generalizes" Lemma 5.2 from alternatives to CNP subdomains.

\footnotetext{
${ }^{11}$ For two integers $k, l \in\{1, \ldots, m\}$, if $k+l \neq m$ and $k+l \neq 2 m$, we define $k \oplus m l:=(k+l) \bmod m$, while if $k+l=m$ or $k+l=2 m$, we define $k \oplus_{m} l:=m$.

${ }^{12}$ This implies that $m_{i}=m_{j}$ for all $i, j \in I$, that the CNP domains $\mathcal{P}_{\mid X_{i}}^{i}$ possess the same factorizations $m_{i}=\prod_{j=1}^{k} q_{j}$ for all $i \in I$ and that the factorization associated with $\mathcal{P}_{\mid Y}^{\prime}$ is $\left(\prod_{j=1}^{k} q_{j}\right) \cdot \# I$.
} 
We claim that there exists an $i \in\{2, \ldots, p\}$ such that $\mathcal{P}_{\mid X_{1}}^{1}$ and $\mathcal{P}_{\mid X_{i}}^{i}$ have identical associated factorizations, and furthermore, $M_{m+1,2 m}(\succ)=X_{i}$ for all $\succ \in \mathcal{P}^{1}$. In addition, there exists a bijection $\varphi_{1, i}: X_{1} \rightarrow X_{i}$ such that $x \in X_{1}$ and $\varphi_{1, i}(x)$ maintain their rank differences in $\mathcal{P}^{1}$. The claim of Substep A implies by Lemmas 5.4 and 5.5 that $\mathcal{P}_{\mid X_{i}}^{1}=\mathcal{P}_{\mid X_{i}}^{i}$ and $m_{1}=\# \mathcal{P}_{\mid X_{1}}^{1}=\# \mathcal{P}_{\mid X_{1} \cup X_{i}}^{1}$. We prove our claim by induction.

Initial step of Substep A: We consider a subdomain $\mathcal{P}^{\prime}$ of $\mathcal{P}^{1}$ with a cyclic permutation domain on top. Note that $\mathcal{P}^{\prime}=\mathcal{P}^{1}$ if $\mathcal{P}_{\mid X_{1}}^{1}$ is a CNP domain of depth 1 . It follows from Lemma 5.4 that there cannot be an alternative $x \in X$ that is ranked by two distinct preferences $\succ$ and $\succ^{\prime}$ in $\mathcal{P}^{\prime}$ at the $m+1$ th position. We shall denote the $n$ distinct alternatives ranked $m$ th by the preferences in $\mathcal{P}^{\prime}$ by $y_{1}, \ldots, y_{n} \in X$, the corresponding preferences by $\succ_{1}, \ldots, \succ_{n}$ and the corresponding top alternatives by $z_{1}, \ldots, z_{n}$, respectively. Let $Y=\left\{y_{1}, \ldots, y_{n}\right\}$ and $Z=\left\{z_{1}, \ldots, z_{n}\right\}$. We can assume without loss of generality that $\mathcal{P}_{\mid Z}^{\prime}=\mathcal{Z}\left(\succ^{*}\right)$, where $z_{1} \succ^{*} z_{2} \succ^{*} \ldots \succ^{*} z_{n}$.

We show that $r k\left[y_{k \oplus_{n} 1}, \succ_{k}\right]=m+2$ for all $k=1, \ldots n$. This assures by Lemma 5.5 that the preferences in $\mathcal{P}^{\prime}$ look like in Table 10. For notational convenience we

Table 10: Substep A

\begin{tabular}{|c|c|c|c|c|}
\hline$\succ_{1}$ & $\succ_{2}$ & $\ldots$ & $\succ_{n-1}$ & $\succ_{n}$ \\
\hline$z_{1}$ & $z_{2}$ & $\ldots$ & $z_{n-1}$ & $z_{n}$ \\
\hline$z_{2}$ & $z_{3}$ & $\ldots$ & $z_{n}$ & $z_{1}$ \\
\hline : & $\vdots$ & $\vdots$ & $\vdots$ & $\vdots$ \\
\hline$z_{n-1}$ & $z_{n}$ & $\ldots$ & $z_{n-3}$ & $z_{n-2}$ \\
\hline$z_{n}$ & $z_{1}$ & $\ldots$ & $z_{n-2}$ & $z_{n-1}$ \\
\hline$\vdots$ & $\vdots$ & & $\vdots$ & $\vdots$ \\
\hline$y_{1}$ & $y_{2}$ & $\ldots$ & $y_{n-1}$ & $y_{n}$ \\
\hline$y_{2}$ & $y_{3}$ & $\cdots$ & $y_{n}$ & $y_{1}$ \\
\hline$\vdots$ & $\begin{array}{l}\vdots \\
.\end{array}$ & $\vdots$ & $\vdots$ & $\vdots$ \\
\hline$y_{n-1}$ & $y_{n}$ & $\ldots$ & $y_{n-3}$ & $y_{n-2}$ \\
\hline$y_{n}$ & $y_{1}$ & $\ldots$ & $y_{n-2}$ & $y_{n-1}$ \\
\hline : & $\vdots$ & & $\vdots$ & $\vdots$ \\
\hline
\end{tabular}

will only show that $r k\left[y_{2}, \succ_{1}\right]=m+2$. We shall denote by $\succ^{\prime}$ a preference with top alternative $y_{2}$. Arguing in an even simpler way than in Lemma 5.4, we can find positive integers $a$ and $b$ such that $y_{2}$ together with at least another alternative from set $X_{1}$ receive the highest Borda scores in profile $\Pi=\left(a \cdot \succ_{2}, b \cdot \succ^{\prime}\right)$ and the lead of $y_{2}$ over the alternatives from $X_{1} \backslash f^{B}(\Pi)$ is at least $m$. Let $U \subseteq X_{1}$ be the set of those alternatives that are ranked higher by $\succ_{1}$ than by $\succ_{2} \cdot{ }^{13}$

Suppose that $f^{B}(\Pi) \cap U \neq \emptyset$. Pick arbitrary alternative $u \in f^{B}(\Pi) \cap U \neq \emptyset$. Then

\footnotetext{
${ }^{13}$ From the structure of $\mathcal{P}_{\mid X_{1}}^{1}$ it follows that $r k\left[u, \succ_{2}\right]-r k\left[u, \succ_{1}\right]=n-1$ for any $u \in U$ and $r k\left[x, \succ_{1}\right]-r k\left[x, \succ_{2}\right]=1$ for any $x \in X_{1} \backslash U$.
} 
there exists a $k \in\{1, \ldots, m / n\}$ such that $r k\left[u, \succ_{2}\right]=k n$. We shall denote by $v \in X_{1}$ the $(k-1) n+1$ th ranked alternative by $\succ_{2} \cdot{ }^{14}$ Let $d=r k\left[y_{2}, \succ_{2}\right]-r k\left[u, \succ_{2}\right]$ and $d^{\prime}=r k\left[u, \succ^{\prime}\right]-r k\left[y_{2}, \succ^{\prime}\right]$. Since $u, y_{2} \in f^{B}(\Pi)$, we must have $a d=b d^{\prime}, u \succ^{\prime} v$ and by Lemma $5.5 r k\left[v, \succ^{\prime}\right]=d^{\prime}+2$. Let us compare the Borda score of $v$ with that of $u$ in $\Pi$. On the one hand $v$ receives $a(n-1)$ points more than $u$ and on the other hand $u$ receives $b$ points more than $v$. Therefore, we must have

$$
a(n-1) \leq b \Leftrightarrow a(n-1) \leq a \frac{d}{d^{\prime}} \Leftrightarrow d^{\prime}(n-1) \leq d ;
$$

a contradiction, since $d<m \leq d^{\prime}$ and $n \geq 2$ by the Assumptions of Step 2. Thus, $f^{B}(\Pi) \cap U=\emptyset$.

Let $z$ be the highest ranked alternative from $f^{B}(\Pi) \backslash\left\{y_{2}\right\}$ by $\succ^{\prime}, \delta=r k\left[y_{2}, \succ_{1}\right]-$ $r k\left[y_{1}, \succ_{1}\right], d=r k\left[y_{2}, \succ_{2}\right]-r k\left[z, \succ_{2}\right]$ and $d^{\prime}=r k\left[z, \succ^{\prime}\right]-r k\left[y_{2}, \succ^{\prime}\right]$. Observe that $z$ has to be the lowest ranked alternative in $\succ_{2}$ from set $f^{B}(\Pi) \backslash\left\{y_{2}\right\}$. Suppose that $\delta \geq 2$, which would mean that $y_{2}$ does not follow immediately $y_{1}$ in $\succ_{1}$. We have to incorporate at least one voter of type $\succ_{1}$ appropriately in order to obtain a contradiction with $\delta \geq 2$. First, we omit a voter of type $\succ_{2}$, which makes $y_{2}$ the single Borda winner with a lead of $d$ over $z$. Second, we compensate this lead by replacing $c=\left\lceil\frac{d}{\delta-1}\right\rceil$ voters of type $\succ_{2}$ with voters of type $\succ_{1}$. If $a \leq c$, then by taking an appropriate multiple of $\Pi$, we can ensure that we have more voters of type $\succ_{2}$ than $c$. Hence, we can assume $a>c$ without loss of generality. Third, we have to take care about not making an alternative $u \in U$ the Borda winning alternative. If $z$ does not lead by $c n$ over alternatives $u \in U$ in $\Pi$, then this can be guaranteed by starting already with an appropriate multiple of $\Pi .^{15}$ Again, we can assume without loss of generality that $a$ and $b$ satisfy this latter requirement. Finally, let $\Pi^{\prime}=\left(c \cdot \succ_{1},(a-c-1) \cdot \succ_{2}, b \cdot \succ^{\prime}\right)$ and $\Pi^{\prime \prime}=\left((a-1) \cdot \succ_{2}, b \cdot \succ^{\prime}\right)$. It can be verified that monotonicity is violated at $z$ by switching from $\Pi^{\prime}$ to $\Pi^{\prime \prime}$, since $z \in f^{B}\left(\Pi^{\prime}\right)$ and $\left\{y_{2}\right\}=f^{B}\left(\Pi^{\prime \prime}\right)$. Thus, we must have $\delta=1$.

Induction hypotheses of Substep A: Assume that we have already obtained a partition $\mathcal{P}^{1,1}, \ldots, \mathcal{P}^{1, t}$ of $\mathcal{P}^{1}$, disjoint subdomains $\mathcal{P}^{2,1}, \ldots, \mathcal{P}^{2, t} \subseteq \mathcal{P} \backslash \mathcal{P}^{1}$ with respective top $n=\frac{m}{t}$ alternatives $X^{j, i}(j=1,2$ and $i=1, \ldots, t)$ such that $t \geq 2, n=$ $\# \mathcal{P}_{[1, n]}^{1, i}=\# \mathcal{P}_{[1, n]}^{2, i}, \mathcal{P}_{[m+1, m+n]}^{1, i}=\mathcal{P}_{[1, n]}^{2, i}$ are CNP domains with associated factorizations $n=q_{1} \ldots q_{l}$ for all $i=1, \ldots, t$ and there exist bijections $\varphi: X^{1, i} \rightarrow X^{2, i}$ satisfying that $x$ and $\varphi_{i}(x)$ maintain their rank differences in $\mathcal{P}^{1, i}$ for all $x \in X^{1, i}$ and all $i=1, \ldots, t{ }^{16}$ This implies that the factorization associated with $\mathcal{P}^{1}$ equals $q_{1} \cdot \ldots \cdot q_{l} \cdot \ldots \cdot q_{l^{\prime}}$ for some $l^{\prime}$ and $q_{l+1}, \ldots, q_{l^{\prime}}$.

Induction step of Substep A: Let $r=q_{l+1}, h=t / r$ and $\mathcal{P}^{j, i}=\left\{\succ_{1}^{j, i}, \ldots, \succ_{n}^{j, i}\right\}$ for all $j=1,2$ and all $i=1, \ldots, t$. We shall denote by $X^{j, i}$ the set of top alternatives of $\mathcal{P}^{j, i}$. If we have labeled the sets $X^{1, i}$ appropriately, then the first $r$ subdomains of $\mathcal{P}_{\mid X_{1}}^{1}$ look like in Table 11. In what follows we shall focus, for notational convenience, on $\mathcal{P}^{1,1}$ and $\mathcal{P}^{1,2}$. In addition, we can assume without loss of generality that the alternatives and preferences are labeled in a way that $r k\left[x_{n}^{1, i}, \succ_{k}^{1,2}\right]=(i-2) n+1+(n-k)$ for

\footnotetext{
${ }^{14}$ Observe that $r k\left[u, \succ_{1}\right]=(k-1) n+1$ and $r k\left[v, \succ_{1}\right]=(k-1) n+2$.

${ }^{15}$ More precisely, we should have first defined $c=\left[\frac{d}{\delta-1}\right]$ and $a, b$ afterwards. However, we have followed a different order for expositional reasons.

${ }^{16}$ Our initial step assured the existence of a partition with $l=1$.
} 
Table 11: Subdomain on top

\begin{tabular}{llll}
$\mathcal{P}^{1,1}$ & $\mathcal{P}^{1,2}$ & $\ldots$ & $\mathcal{P}^{1, r}$ \\
\hline$X^{1,1}$ & $X^{1,2}$ & $\ldots$ & $X^{1, r}$ \\
$X^{1,2}$ & $X^{1,3}$ & $\ldots$ & $X^{1,1}$ \\
$\vdots$ & $\vdots$ & & $\vdots$ \\
$X^{1, r}$ & $X^{1,1}$ & $\ldots$ & $X^{1, r-1}$ \\
$\vdots$ & $\vdots$ & & $\vdots$ \\
$X^{1, t-r+1}$ & $X^{1, t-r+2}$ & $\ldots$ & $X^{1, t}$ \\
$X^{1, t-r+2}$ & $X^{1, t-r+3}$ & $\ldots$ & $X^{1, t-r+1}$ \\
$\vdots$ & $\vdots$ & & $\vdots$ \\
$X^{1, t}$ & $X^{1, t-r+1}$ & $\ldots$ & $X^{1, t-1}$ \\
$\vdots$ & $\vdots$ & & $\vdots$
\end{tabular}

all $k=1, \ldots, n$ and all $i=2, \ldots, t$ for which $i-1$ is not divisible by $r$, and otherwise, $r k\left[x_{n}^{1, i}, \succ_{k}^{1,2}\right]=(i+r-2) n+1+(n-k)$ for all $k=1, \ldots, n$ and all $i=1, \ldots, t$.

We shall denote by $y_{1}, \ldots, y_{n}$ the $n$ distinct alternatives ranked $m+1$ th by the preferences $\succ_{1}^{1,2}, \ldots, \succ_{n}^{1,2}$, respectively. Let $Y=\left\{y_{1}, \ldots, y_{n}\right\}$. Moreover, for all $i=$ $1, \ldots, n$ we simply write $\succ_{i}, \succ_{i}^{\prime}$ and $\succ_{i}^{\prime \prime}$ for $\succ_{i}^{1,1}, \succ_{i}^{1,2}$ and $\succ_{i}^{2,2}$, respectively.

We can find positive integers $a$ and $b$ such that $Y$ and at least a set of alternatives $X^{\prime} \subseteq X_{1}$ receives the highest Borda score in profile $\Pi=\left(a \cdot \succ_{1}^{\prime}, \ldots, a \cdot \succ_{n}^{\prime}, b \cdot \succ_{1}^{\prime \prime}\right.$ $\left., \ldots, b \cdot \succ_{n}^{\prime \prime}\right)$. Let $U \subseteq X_{1}$ be the set of those alternatives that are ranked higher by $\succ_{1}$ than by $\succ_{1}^{\prime}$. Observe that $U=\cup_{i=0}^{h-1} X^{1, i r+1}$.

Suppose that there exists an $i=1, \ldots, h$ such that $u \in f^{B}(\Pi) \cap X^{1,(i-1) r+1}$. Pick an arbitrary alternative $y \in Y$. Since $\{u, y\} \in f^{B}(\Pi)$, we must have $a[(h-i) r+1] n^{2}=b d^{\prime}$, where $d^{\prime}=\sum_{i=1}^{n} r k\left[u, \succ_{i}^{\prime \prime}\right]-r k\left[y, \succ_{i}^{\prime \prime}\right]$. Take an alternative $v$ from $X^{1,(i-1) r+2}$ such that $d^{\prime \prime}=\sum_{i=1}^{n} r k\left[v, \succ_{i}^{\prime \prime}\right]-r k\left[y, \succ_{i}^{\prime \prime}\right]$ is as small as possible. Since the Borda score of $v$ cannot be greater than that of $u$ in $\Pi$, we must have $a n(r-1) n \leq b\left(d^{\prime \prime}-d^{\prime}\right)$. Therefore,

$$
\frac{b d^{\prime}}{[(h-i) r+1] n^{2}} n^{2}(r-1) \leq b\left(d^{\prime \prime}-d^{\prime}\right) \Leftrightarrow d^{\prime}(r-1) \leq\left(d^{\prime \prime}-d^{\prime}\right)[(h-i) r+1] .
$$

By the hypothesis of Step 2 and by Lemmas 5.4 and 5.5 we must have $d^{\prime} \geq m n=t n^{2}$. The value $d^{\prime \prime}-d^{\prime}$ would be the largest if $u$ is ranked higher than $v$ by any preference $\succ_{i}^{\prime \prime} \in$ $\mathcal{P}^{2,2}$. Then the alternatives from $X^{1,(i-1) r+2}$ must follow immediately the alternatives from $X^{1,(i-1) r+1}$ in any $\succ_{i}^{\prime \prime} \in \mathcal{P}^{2,2}$ by Lemma 5.5. Moreover, we have $d^{\prime \prime}-d^{\prime} \leq n^{2}$ by Lemma 5.5 and by the choice of $v$, which together with equation (6.1) implies

$$
t n^{2} \leq d^{\prime}(r-1) \leq\left(d^{\prime \prime}-d^{\prime}\right)[(h-i) r+1] \leq n^{2}[(h-i) r+1] .
$$

It follows from these inequalities that $r h=t \leq[(h-i) r+1]$, which implies $i r \leq 1$. Therefore, since $r, n \geq 2, h \geq 1$ and $i \geq 1$ we obtained a contradiction and we conclude that $f^{B}(\Pi) \cap U=\emptyset$.

Define $v=\max \left\{i=1, \ldots, t \mid X^{1, i} \cap f^{B}(\Pi) \neq \emptyset\right\}$ and pick an alternative $z$ from $X^{1, v} \cap f^{B}(\Pi)$. Let $\succ^{\prime} \in \mathcal{P}^{1,2}$ the preference that ranks $z$ highest. For notational 
convenience we can assume that $\succ^{\prime}=\succ_{n}^{\prime}$ and $\succ^{\prime}$ ranks $y_{n}$ as the highest alternative from $Y{ }^{17}$ Hence, $z$ is the highest ranked alternative from $X^{1, v}$ by $\succ_{n}^{\prime}$. Observe that from the way how we labeled the alternatives of $X_{1}$ and our assumption on $\varphi: X^{1,2} \rightarrow X^{2,2}=Y$ it follows that $r k\left[y_{n}, \succ_{k}^{\prime}\right]-r k\left[x_{n}^{1,2}, \succ_{k}^{\prime}\right]=m$ for all $k=1, \ldots, n$. In addition, we can assume for notational convenience that $\succ_{k \mid X^{1, v}}=\succ_{k \mid X^{1, v}}^{\prime}$ for all $k=1, \ldots, n$. We will show that $r k\left[y_{n}, \succ_{k}\right]=m+n+1+(n-k)$ for all $k=1, \ldots, n$. Observe that $r k\left[y_{n}, \succ_{k}\right]>m+n$, since the shortest sequence of alternatives that must follow an already prescribed order is of length $n$ and by Lemma 5.4 none of the alternatives of $Y$ can be ranked $m+1$ th by a preference of $\mathcal{P}^{1,1}$. Now take an arbitrary index $k=1, \ldots, n$ and let $\delta_{k}=r k\left[y_{n}, \succ_{k}\right]-(m+1+n-k)$.

Suppose that $\delta_{k}>n$. By replacing a preference $\succ_{1}^{\prime}$ with $\succ_{n}^{\prime}$ in $\Pi$, we can achieve that $f^{B}(\Pi)$ contains only $y_{n}$ from $Y$ and only $z$ from $X^{1, v}$. In what follows we shall denote this modified profile by $\Pi$ with a slight abuse of notation. Let $d=r k\left[y_{n}, \succ_{n}^{\prime}\right]-$ $r k\left[z, \succ_{n}^{\prime}\right]$ and $d^{\prime}=\sum_{i=1}^{n} r k\left[z, \succ_{i}^{\prime \prime}\right]-r k\left[y_{n}, \succ_{i}^{\prime \prime}\right]$. Note that $d^{\prime} \geq m n$ by the assumptions of Step 2 and by Lemmas 5.4 and 5.5. Now we have to incorporate at least one voter of type $\succ_{k}$ into $\Pi$ in order to obtain a contradiction with $\delta_{k}>n$. First, we omit a voter of type $\succ_{1}^{\prime}$, which makes $y_{n}$ the single Borda winner with a lead of $d$ over $z$. Second, we compensate this lead by replacing $c=\left\lceil\frac{d}{\delta_{k}-n}\right\rceil$ voters of type $\succ_{k}^{\prime}$ with voters of type $\succ_{k}$. If $a \leq c$, then by starting with an appropriate multiple of $\Pi$, we can ensure that we have more than $c$ voters of type $\succ_{k}^{\prime}$. Hence, we can assume $a>c$ without loss of generality. Third, we have to take care about not making an alternative $u \in U$ the Borda winning alternative. If $z$ does not lead by $\mathrm{cm}$ over alternatives $u \in U$ in $\Pi$, then this can be guaranteed by starting already with an appropriate multiple of $\Pi{ }^{18}$ Thus, we can assume without loss of generality that $a$ and $b$ satisfy this latter requirement. Let $\Pi^{\prime}=\left((a-2) \cdot \succ_{1}^{\prime}, a \cdot \succ_{2}^{\prime}, \ldots, a \cdot \succ_{k-1}^{\prime}, c \cdot \succ_{k},(a-c) \cdot \succ_{k}^{\prime}, a \cdot \succ_{k+1}^{\prime}\right.$ $\left., \ldots, a \cdot \succ_{n-1}^{\prime},(a+1) \cdot \succ_{n}^{\prime}, b \cdot \succ_{1}^{\prime \prime}, \ldots, b \cdot \succ_{n}^{\prime \prime}\right)$ and $\Pi^{\prime \prime}=\left((a-2) \cdot \succ_{1}^{\prime}, a \cdot \succ_{2}^{\prime}, \ldots, a \cdot \succ_{n-1}^{\prime}\right.$ $\left.,(a+1) \cdot \succ_{n}^{\prime}, b \cdot \succ_{1}^{\prime \prime}, \ldots, b \cdot \succ_{n}^{\prime \prime}\right)$. It can be verified that monotonicity is violated at $z$ by switching from $\Pi^{\prime}$ to $\Pi^{\prime \prime}$, since $\{z\}=f^{B}\left(\Pi^{\prime}\right)$ and $\left\{y_{n}\right\}=f^{B}\left(\Pi^{\prime \prime}\right)$. Thus, we cannot have $\delta_{k}>n$.

Suppose that $\delta_{k}<n$ for some $k$. Then alternative $y_{n}$ has to be ranked by at least two different preferences of $\mathcal{P}^{1,1}$ at the same position, since $\delta_{k} \leq n$ for all $i=1, \ldots, n$. However, this is in contradiction with Lemmas 5.4 and 5.5. Hence, we must have $\delta_{k}=n$ for all $k=1, \ldots, n$.

Therefore, since the shortest sequence of alternatives that must follow an already prescribed order is of length $n$ and by Lemma 5.5 none of the alternatives of $Y$ can be ranked $m+1$ th by a preference of $\mathcal{P}^{1,1}$, we obtained that $n=\# \mathcal{P}_{\mid X_{1} \cup Y}^{1,2}$ and $\mathcal{P}_{[m+n+1, m+2 n]}^{1,1}=\mathcal{P}_{[1, n]}^{2,2}$. Thus, the domain of Table 11 extends to a domain as illustrated in Table 12. Now Lemma 5.5 implies that the alternatives $\cup_{i=1}^{r} Y_{i}$ must form a CNP domain of depth $l+1$ with an associated factorization $\prod_{i=1}^{l+1} q_{i}$. Therefore, our induction works and the induction hypothesis is true for depth $l+1$. Arriving to $l^{\prime}$, we see that the claim of Substep A is true, since it follows from Lemma 5.5 that there exists an $i=2, \ldots, p$ for which $\mathcal{P}^{2,1}, \ldots, \mathcal{P}^{2, t}$ partitions $\mathcal{P}^{i}$.

Substep B: We "generalize" Lemma 5.3 from alternatives to CNP subdomains on

\footnotetext{
${ }^{17}$ Otherwise, we would relabel the alternatives of $X_{1}$ and $Y$ as well as the preferences of $\mathcal{P}^{1,2}$.

${ }^{18}$ More precisely, we should have first defined $c=\left\lceil\frac{d}{\delta_{k}-n}\right\rceil$ and $a, b$ afterwards. Again, we have followed a different order for expositional reasons.
} 
Table 12: Extended domain

\begin{tabular}{llll}
$\mathcal{P}^{1,1}$ & $\mathcal{P}^{1,2}$ & $\ldots$ & $\mathcal{P}^{1, r}$ \\
\hline$X^{1,1}$ & $X^{1,2}$ & $\ldots$ & $X^{1, r}$ \\
$X^{1,2}$ & $X^{1,3}$ & $\ldots$ & $X^{1,1}$ \\
$\vdots$ & $\vdots$ & & $\vdots$ \\
$X^{1, r}$ & $X^{1,1}$ & $\ldots$ & $X^{1, r-1}$ \\
$\vdots$ & $\vdots$ & & $\vdots$ \\
$X^{1, t-r+1}$ & $X^{1, t-r+2}$ & $\ldots$ & $X^{1, t}$ \\
$X^{1, t-r+2}$ & $X^{1, t-r+3}$ & $\ldots$ & $X^{1, t-r+1}$ \\
$\vdots$ & $\vdots$ & & $\vdots$ \\
$X^{1, t}$ & $X^{1, t-r+1}$ & $\ldots$ & $X^{1, t-1}$ \\
$Y_{1}$ & $Y_{2}$ & $\ldots$ & $Y_{r}$ \\
$Y_{2}$ & $Y_{3}$ & $\ldots$ & $Y_{1}$ \\
$\vdots$ & $\vdots$ & & $\vdots$
\end{tabular}

top. Informally, we show that subdomains with two consecutive CNP domains on top form cycles of CNP domains on top.

Substep A implies that there exists an $I \subseteq\{1, \ldots, p\}$ such that for all $i \in I$ the subdomains $\mathcal{P}_{\mid X_{i}}^{i}$ have all identical factorizations, there exists a $j \in I \backslash\{i\}$ for which $M_{m+1,2 m}(\succ)=X_{j}$ for all $\succ \in \mathcal{P}^{i}$ and there exists a bijection $\varphi_{i, j}: X_{i} \rightarrow X_{j}$ such that $x \in X_{i}$ and $\varphi_{i, j}(x)$ maintain their rank differences in $\mathcal{P}^{i}$. We shall assume for notational convenience that $I=\{1, \ldots, r\}$. Hence, there exists a $\sigma:\{1, \ldots, r\} \rightarrow\{1, \ldots, r\}$ telling us, which set $X_{\sigma(i)}$ of alternatives must follow immediately the top set $X_{i}$ of alternatives for all $i=1, \ldots, r$. In Substep B we demonstrate that $\sigma$ is a bijection. This is clearly the case if $r=2$.

Thus, we can assume that $r>2$. Our proof will be similar to that of Lemmas 5.1-5.3, but we have to replace the alternatives appearing in those proofs with "nested cycles", which will complicate the argument.

Suppose that $\sigma$ does not define a bijection. Then there exists $i, i^{\prime}, i^{\prime \prime} \in\{1, \ldots r\}$ such that $i \neq i^{\prime}, i \neq i^{\prime \prime}, i^{\prime} \neq i^{\prime \prime}, \sigma\left(i^{\prime}\right)=i$ and $\sigma\left(i^{\prime \prime}\right)=i$. Moreover, $m=m_{i}=$ $m_{i^{\prime}}=m_{i^{\prime \prime}}$ by Substep A. For notational convenience let $X^{\prime}=\left\{x_{1}, \ldots, x_{m}\right\}=X_{i}, Y=$ $\left\{y_{1}, \ldots, y_{m}\right\}=X_{i^{\prime}}, Z=\left\{z_{1}, \ldots, z_{m}\right\}=X_{i^{\prime \prime}}$ such that $\varphi_{i^{\prime}, i}\left(y_{l}\right)=x_{l}$ and $\varphi_{i^{\prime \prime}, i}\left(z_{l}\right)=x_{l}$ for all $l=1, \ldots, m$. Pick preferences $\succ_{1}, \ldots, \succ_{m}, \succ_{1}^{\prime}, \ldots, \succ_{m}^{\prime}, \succ_{1}^{\prime \prime}, \ldots, \succ_{m}^{\prime \prime} \in \mathcal{P}$ with respective top alternatives $x_{1}, \ldots, x_{m}, y_{1}, \ldots, y_{m}, z_{1}, \ldots, z_{m}$. If $r=3$, then the alternatives from $X^{\prime}$ cannot be lowest ranked alternatives; a contradiction. If $r>3$, then we can assume the existence of a preference $\succ \in \mathcal{P}$ in which the alternatives from $Y$ are ranked above the alternatives from $Z$ and the alternatives from $Z$ are ranked above the alternatives from $X^{\prime}$ by part (ii) of the minimal richness condition. For notational convenience we can assume that

$$
\begin{aligned}
& y_{l} \succ_{l}^{\prime} \ldots \succ_{l}^{\prime} y_{l^{\prime}} \succ_{l}^{\prime} x_{1} \succ_{l}^{\prime} \ldots \succ_{l}^{\prime} x_{m} \succ_{l}^{\prime} \ldots \\
& z_{k} \succ_{k}^{\prime \prime} \ldots \succ_{k}^{\prime \prime} z_{k^{\prime}} \succ_{k}^{\prime \prime} x_{1} \succ_{k}^{\prime \prime} \ldots \succ_{k}^{\prime \prime} x_{m} \succ_{k}^{\prime \prime} \ldots
\end{aligned}
$$


and

$$
\ldots \succ y_{s} \succ \ldots \succ y_{s^{\prime}} \succ \ldots \succ z_{t} \succ \ldots \succ z_{t^{\prime}} \succ \ldots \succ x_{1} \succ \ldots \succ x_{m}
$$

for some $l, l^{\prime}, k, k^{\prime}, s, s^{\prime}, t, t^{\prime} \in\{1, \ldots, m\}$.

Let $J=\arg \min _{j \in\{1, \ldots, m\}} \sum_{u=1}^{m} r k\left[y_{j}, \succ_{u}\right]$ and $Y^{\prime}=\left\{y_{j} \in Y \mid j \in J\right\}$. Then there exist positive integers $a$ and $b$ such that $b>m^{2}+1$ and that profile

$$
\Pi=\left(a \cdot \succ_{1}, \ldots, a \cdot \succ_{m}, b \cdot \succ_{1}^{\prime}, \ldots, b \cdot \succ_{m}^{\prime}\right),
$$

has alternatives $Y^{\prime} \cup X^{\prime}$ indifferent on top with a lead of at least $\left(m^{2}+1\right) q$ over the alternatives from $X \backslash\left(X^{\prime} \cup Y\right)$. We consider profile

$$
\Pi^{\prime}=\left(a \cdot \succ_{1}, \ldots, a \cdot \succ_{m},(b-1) \cdot \succ_{1}^{\prime}, \ldots,(b-1) \cdot \succ_{m}^{\prime}\right)
$$

in which the top alternatives $X^{\prime}$ have a lead of $m^{2}$ over alternatives $Y^{\prime}$. To obtain $\Pi^{\prime \prime}$ from $\Pi^{\prime}$ we replace $m^{2}+1$ preferences of type $\succ_{s}^{\prime}$ with $m^{2}+1$ preferences of type $\succ$. It can be verified that $f^{B}\left(\Pi^{\prime}\right)=X^{\prime}$ and $f^{B}\left(\Pi^{\prime \prime}\right)=Y^{\prime}$. Thus, we have a violation of monotonicity at any alternative $y \in Y^{\prime}$ if we switch from $\Pi^{\prime \prime}$ to $\Pi^{\prime}$.

Substep C: What Step 1 showed for alternatives is now established for subdomains with CNP domains on top: the union of some subdomains with CNP domains of identical factorization on top form a subdomain with a deeper CNP domain on top, which completes the proof of Step 2.

Substep B established that the cycles of permutation $\sigma$ partition $\{1, \ldots, r\}$ into sets $I_{1}, \ldots, I_{s}$. In what follows we consider, for notational convenience, the case of $I=I_{1}=\{1, \ldots, k\}$ and $\sigma(1)=2, \ldots, \sigma(k-1)=k, \sigma(k)=1$. First, in an analogous way to Step 1 we show in Substep $C$ that the cycles formed by alternatives $X_{1}, \ldots, X_{k}$ follow each other in a cyclic pattern in $\mathcal{P}^{1} \cup \ldots \cup \mathcal{P}^{k}$; that is, for all $i=1, \ldots, k$ we have in $\mathcal{P}^{i}$ that the alternatives from $X_{i \oplus_{k} 1}$ follow those from $X_{i}$, the alternatives from $X_{i \oplus_{k} 2}$ follow those from $X_{i \oplus_{k} 1}$, and so on. Second, we claim that there exist bijections $\tau_{i, j}: X_{i} \rightarrow X_{j}(i, j=1, \ldots, k)$ such that

$$
\left[x \in X_{i}, v=\tau_{i, j}(x), x \succ v, x \succ^{\prime} v\right] \Rightarrow r k[v, \succ]-r k[x, \succ]=r k\left[v, \succ^{\prime}\right]-r k\left[x, \succ^{\prime}\right]
$$

for all $\succ, \succ^{\prime} \in \mathcal{P}^{1} \cup \ldots \cup \mathcal{P}^{k}$ and all $i \neq j, i, j=1, \ldots, k$.

Clearly, both claims are true for the case of $k \leq 2$. Hence, we can assume that $k \geq 3$. We know by Substep B that the first claim of Substep C is true for the top $2 m$ alternatives of any preferences in $\mathcal{P}^{i}$ and that we can define bijections $\tau_{i, i \oplus_{k} 1}: X_{i} \rightarrow$ $X_{i \oplus_{k} 1}$ in a way that equation (6.2) holds true for all $i=1, \ldots, k$ if we restrict ourselves to the top $2 m$ alternatives of $\mathcal{P}^{1} \cup \ldots \cup \mathcal{P}^{k}$.

Our induction hypotheses is that the claim holds true for the top $l m$ alternatives, where $l \in\{2, \ldots, k-1\}$, of any preference in $\mathcal{P}_{1} \cup \ldots \cup \mathcal{P}_{k} \cdot{ }^{19}$ For purely notational convenience let $Y=\left\{y_{1}, \ldots, y_{m}\right\}=X_{l}, Z=\left\{z_{1}, \ldots, z_{m}\right\}=X_{l+1}$, and $\tau_{l, l+1}\left(y_{i}\right)=z_{i}$ for all $i=1, \ldots, m$. We will just consider the case of $z_{1}$. The other elements of $Z$ can be handled in the same way. We shall denote by $\succ \in \mathcal{P}^{1}$ the preference that ranks $y_{1}$ as the highest ranked $Y$ alternative; i.e., $r k\left[y_{1}, \succ\right]=m(l-1)+1$. Moreover, let $\succ^{\prime}$ be a preference ranking $y_{1}$ on the top and let $\succ^{\prime \prime}$ be a preference ranking $z_{1}$ on

\footnotetext{
${ }^{19}$ This includes that we have defined bijections $\tau_{i, i \oplus_{k} l-1}: X_{i} \rightarrow X_{i \oplus_{k} l-1}$ in a way that equation
} (6.2) holds true for all $i=1, \ldots, k$ if we restrict ourselves to the top $l m$ alternatives of $\mathcal{P}^{1} \cup \ldots \cup \mathcal{P}^{k}$. 
the top. There exists positive integers $a$ and $b$ such that profile $\Pi=\left(a \cdot \succ^{\prime}, b \cdot \succ^{\prime \prime}\right)$ has alternatives $z_{1}$ and $Y^{\prime} \subseteq Y$ on the top. Let $y_{s}=\max \left\{t \in\{1, \ldots, m\} \mid y_{t} \in Y^{\prime}\right\}$.

Suppose that alternative $z_{1}$ does not immediately follow $y_{m}$ in $\succ$; i.e, $r k\left[z_{1}, \succ\right]>$ $m l+1$. Let $\delta=r k\left[z_{1}, \succ\right]-(m l+1), d=r k\left[z_{1}, \succ^{\prime}\right]-r k\left[y_{s}, \succ^{\prime}\right]$ and $c=\left[\frac{d}{\delta}\right]$. Then considering profiles $\Pi^{\prime}=\left((a-1) \cdot \succ^{\prime}, b \cdot \succ^{\prime \prime}\right)$ and $\Pi^{\prime \prime}=\left(c \cdot \succ,(a-c-1) \cdot \succ^{\prime}, b \cdot \succ^{\prime \prime}\right)$, we can verify that $y_{s} \in f^{B}\left(\Pi^{\prime \prime}\right)$ and $f^{B}\left(\Pi^{\prime}\right)=\left\{z_{1}\right\}$ if $a$ and $b$ were selected large enough so that no other alternative can interfere and $a>c+1$. Monotonicity is now violated at $y_{s}$ if we switch from $\Pi^{\prime \prime}$ to $\Pi^{\prime}$.

It follows from the above defined $\tau_{l, l+1}: X_{l} \rightarrow X_{l+1}, \tau_{1, l}: X_{1} \rightarrow X_{l}$ and transitivity that we obtained a bijection $\tau_{1, l+1}: X_{1} \rightarrow X_{l+1}$ in a way that equation (6.2) holds true for all $l=1, \ldots, k-1$ if we restrict ourselves to the top $(l+1) m$ alternatives of $\mathcal{P}^{1} \cup \ldots \cup \mathcal{P}^{k}$. One can obtain the remaining bijections in an analogous way.

We conclude that we have constructed the required sets $Y=\cup_{i=1}^{k} X_{i}$ and $\mathcal{P}^{\prime}=$ $\cup_{i=1}^{k} \mathcal{P}^{i}$ by induction.

Step 3: The partition $X_{1}, \ldots, X_{p}$ of $X$ in Step 1 satisfies the requirements of Step 2. Finally, it follows by induction from Step 2 that $\mathcal{P}$ has to be a CNP domain. 


\section{References}

[1] Barbie, M., Puppe, C. and Tasnádi, A. (2006), "Non-Manipulable Domains for the Borda Count," Economic Theory 27, 411-430.

[2] Black, D. (1948), "On the Rationale of Group Decision Making," Jornal of Political Economy 56, 23-34.

[3] Bochet, O. and Storcken, T. (2005), "Maximal Domains for Strategy-Proof or Maskin Monotonic Choice Rules," mimeographed.

[4] Erdem, O. and Sanver, R. (2005), "Minimal Monotonic Extensions of Scoring Rules," Social Choice 83 Welfare 25, 31-42.

[5] Gaertner, W. (2001), Domain Conditions in Social Choice Theory, Cambridge University Press.

[6] Kalai, E. and Muller, E. (1977), "Characterization of Domains Admitting Nondictatorial Social Welfare Functions and Nonmanipulable Voting Procedures," Journal of Economic Theory 16, 457-469.

[7] Kalai, E. and Ritz, Z. (1980), "Characterization of the Private Alternatives Domains Admitting Arrow Social Welfare Functions," Journal of Economic Theory $22,23-36$.

[8] Maskin, E. (1999), "Nash Equilibrium and Welfare Optimality," Review of Economic Studies, 66, 23-38.

[9] Muller, E. and Satterthwaite, M.A. (1977), "The Equivalence of Strong Positive Association and Strategy-Proofness," Journal of Economic Theory 14, 412-418.

[10] Sanver, R. (2007a), "Nash Implementability of the Plurality Rule over Restricted Domains," Economics Letters, forthcoming.

[11] Sanver, R. (2007b), "Strategy-Proofness of the Plurality Rule over Restricted Domains," mimeographed. 